\title{
Influential Article Review - Formulating a Healthy Ageing at Workplace Scale: A Unique Approach to Evaluating Professional Growth
}

\author{
Jamal Albert \\ Cecilia Pearson \\ Sienna-Rose Daly
}

This paper examines leadership and development. We present insights from a highly influential paper. Here are the highlights from this paper: The aging of the world's population has become a grand societal challenge, which requires an urgent response from researchers, professionals, and the wider society. One way to respond to this challenge is by supporting individuals in sustaining longer and healthier working lives, that is, age successfully. Although the advantages of promoting and enabling successful aging at work have been widely acknowledged, no single instrument for measuring this construct has been published so far. We develop and test in three consecutive samples a two-dimensional successful aging at work scale. This novel instrument is rooted conceptually in the most recent theoretical developments and is applicable to a variety of work settings and groups of workers. It can be used by both employees and employers for the purposes of individual and organizational development. We also propose directions for future research. For our overseas readers, we then present the insights from this paper in Spanish, French, Portuguese, and German.

Keywords: successful aging at work, growth, development, lifespan, scale, measurement

\section{SUMMARY}

- We developed and established the psychometric properties of the first single measure for SAW in the contemporary meaning of this construct. We view SAW as a developmental process that may occur at all career stages and can be captured through individual-level outcomes such as improved WA and well-being. We operationalized the construct SAW through two clusters of employees' perceptions: individuals' psychological experiences of age-related growth in the workplace, and

- Strategies for achieving SAW. Experiences of growth may refer to various areas of perceived agerelated positive changes, among which are improved knowledge and experience, social skills, emotion regulation, and adaptability in work-related situations. Strategies for SAW may include both employee and organizational behaviors.

- Subsequent empirical analysis with data from three consecutive MTurk samples with overall 417 workers in organizational setting revealed that the employees' experiences of SAW largely overlapped with the employees' perceptions of individual-level facilitators of SAW. 
- This study contributed to theory development in several ways. First, it systemized and further conceptualized key domains of the emerging construct SAW by linking person and environmental variables. Thus, it responded to recent calls for integrating multilevel factors when examining this construct. Second, it focused on the synergies between these domains and gained new insights into how they operate together, as well as separately. Third, it translated into practical terms each domain and explored its construct and criterion-related validity with regard to other, well-known constructs and demographic variables.

- Future research needs to provide further evidence of the validity of the SAW scale. For example, we found a significant positive relationship of age with organizational tenure, but we did not find evidence of age-related differences regarding the employees' self-perceptions of SAW.

- The lack of evidence of age-related differences may be because the age characteristics of our participant groups were not specified well enough or because generally there is no clear, definitive criterion for dividing workers into «older» and «younger».

- 60 years of age. It may be the case that people aged 60 years and over, who stay in the workforce, experience SAW differently compared to workers at younger ages. Future research should address this issue. In addition, it is possible that various moderators play a role in workers' aging. Importantly, the lack of evidence of age-related differences in the employees' self-perceptions of SAW may indicate that the measure, which we suggest, captures SAW at all career stages.

\section{HIGHLY INFLUENTIAL ARTICLE}

We used the following article as a basis of our evaluation:

Taneva, S., \& Yankov, G. (2020). A New Perspective to Measuring Employee Growth: Developing a Successful Aging at Work Scale. Personnel Assessment and Decisions, 6(1).

This is the link to the publisher's website: https://scholarworks.bgsu.edu/pad/vol6/iss1/5/

\section{INTRODUCTION}

The emerging concept of successful aging at work (SAW) is rooted in the contemporary interpretations of the organizational and lifespan developmental theories. It emphasizes the important role of workplaces for successful aging (Olson \& Schultz, 2019), implying that long and positive work-related experiences can be sustained through successful adaptation to both intra-individual (e.g., age-related) and external (e.g., environmental) changes (Kooij, 2015; Olson \& Schultz, 2019; Zacher, 2015a). Kooij (2015) described SAW as the sustainable maintenance of an individual's work ability, health, and motivation across the working life cycle. In this definition, the active role of the employee for the creation of work resources and securing an ongoing person-environment fit is of key importance. Zacher (2015a, b) pointed out that SAW is not limited to maintaining current levels of functioning and may refer to a worker's development or growth. Thus, SAW can be viewed as an intra-individual age-related trajectory to a work outcome (e.g., well-being or job performance) that deviates positively from the average age-related trajectory. Even an employee who has experienced a decline in a work outcome over time may be aging successfully at work when his/her trajectory is more positive than the average age-related trajectory (Zacher, 2015a, b).

In a systematic way, Zacher, Kooij, and Beier (2018) outlined the factors contributing to active aging at work (AAW), which is a construct that somewhat overlaps with SAW (cf. Zacher, 2015a). AAW is described as workers' ability to "maintain or improve (1) their physical, mental, and social well-being; (2) continue to show high levels of work engagement and performance; and (3) experience fair treatment and employment security" in the aging process (Zacher et al., 2018, p. 37). Because development is a lifelong process, AAW refers to workers of all ages and career stages. Multilevel factors (individual, team, organizational, and wider societal) contribute to AAW/SAW (see also Kooij, Zacher, Wang, \& 
Heckhausen, 2019). The idea of understanding AAW/ SAW in relation to both internal (e.g., person) and external (e.g., environmental) factors is in line with the ongoing criticism that being focused mostly on the process of intra-individual development, lifespan theories overall are likely to neglect the effects of the context on the individual's development (Olson \& Schultz, 2019).

Given the increasing interest in AAW/SAW from a contemporary lifespan perspective (Zacher et al., 2018), it is surprising that no measure of this construct has been developed yet. In two consecutive studies in 2006 and 2007, Robson and his colleagues conceptualized tentative criteria for successful aging in the workplace and developed an inventory for measuring them (Robson \& Hansson, 2007; Robson, Hansson, Abalos, \& Booth, 2006). Zacher \& Rudolph (2017) criticized this model for neglecting the contextual factors and work outcomes by focusing entirely on person factors (behavioral strategies). In addition, Zacher \& Rudolph (2017) concluded that because the behavioral strategies suggested by Robson et al. (Robson \& Hansson, 2007; Robson et al., 2006) do not interact with age, these strategies cannot be considered successful aging strategies. Most recently, Kooij and colleagues acknowledged the difficulty of operationalizing and, thus, measuring the construct SAW (Kooij et al., 2019).As with Zacher (2015a), we understand SAW as a process of multidimensional intra-individual growth, which is age related, associated with various subjective and objective criteria, and may be facilitated (or constrained) by multilevel factors. Based on Zacher's (2015a) definition of SAW and the framework of AAW factors by Zacher et al. (2018), the purpose of our study is to extend the knowledge of SAW by: (a) operationalizing important facets of the construct SAW and (b) creating a single measure of SAW. We focus on the individual experiences and outcomes of SAW, as well as on both person and environmental antecedents of SAW in organizational context. We also consider the potential interactions of these factors with workers' age (cf. Zacher et al., 2015a, 2018). We test the psychometric properties of the new measure in three consecutive MTurk samples and suggest directions for future research.

\section{Operationalizing Successful Aging at Work}

Employees' Experiences of SAW

The impacts of age-related changes on work behaviors and outcomes (especially in late career) are extensively documented. The nature and extent of age-related changes may vary significantly across individuals due to differences in person and environmental factors. These changes (e.g., in workers' cognitive abilities, personality, and motivation) are likely to reflect various developmental losses and gains across the working life cycle (Kanfer \& Ackerman, 2004; Warr, 2001). Recently, the number of studies focused on the positive (i.e., developmental gains) as opposed to loss-related (i.e., developmental declines) aspects of the aging process has increased. For example, in a qualitative study with 37 older workers (aged 55 years and over) from two countries (Bulgaria and United Kingdom) and two industrial sectors (healthcare and information and communication technologies), Taneva, Arnold and Nicolson (2016) found that late career workers are well aware of how age-related changes may affect their work life and outcomes. Importantly, workers associate some of these changes (e.g., increased knowledge and experience, enhanced social and emotional experience) with their personal and professional development and, ultimately, with improved work outcomes. Thus, workers' perceptions of positive age-related changes (developmental gains; cf. Kanfer \& Ackerman, 2004) are likely to indicate workers' experiences of SAW.

In Taneva et al.'s (2016) study, along with their perceptions of increased knowledge and experience, (older) workers communicated perceptions of other areas of personal and professional growth, among which increased self-awareness and confidence, improved social skills, emotion regulation, ability to solve complex problems, and general adaptation to contextual changes. These findings, extracted from workers' narratives, correspond with the outcomes of earlier research in the lifespan tradition (e.g., Carstensen et al., 2011; Kanfer \& Ackerman, 2004). We suggest that workers' conceptualizations of positive intra-individual changes may shape an important domain of the construct SAW.

\section{Personal and Organizational Strategies for SAW}

From a lifespan theory perspective, aging is a proactive process of adaptation, driven by self-regulation in the form of life management strategies that individuals apply in order to deal with age-related changes 
(e.g., loss, gain, and/ or reorganization of resources) in themselves as well as changes in their environment (Kanfer \& Ackerman, 2004). The selective optimization with compensation (SOC) model (Baltes \& Baltes, 1990) proposes that individuals are likely to use strategies that fall into three categories of self-regulation processes (selection, optimization, and compensation) in their attempts to adapt to both age-related changes and changes in their environment. These proactive behaviors become more important with increased age and can be particularly useful in the workplace. Starting with the pioneering work of Abraham and Hansson (1995), a plethora of studies has demonstrated the positive effects of SOC strategies on (older) workers' well-being and performance (e.g., Moghimi, Scheibe, \& Freund, 2019; Moghimi, Zacher, Shreible, \& Van Yperen, 2017). Thus, SOC strategies may be an effective mechanism for achieving SAW.

Combining the advantages of the job design and lifespan theories, Truxillo, Cadiz, Rineer, Zaniboni, and Fraccaroli, (2012) proposed potential age-related effects of three subgroups of job characteristics (task, knowledge, and social) on work engagement, job satisfaction, and job performance. For example, they suggested that due to intra-individual age-related changes, some job characteristics such as job autonomy, task significance, skill variety, and social support may be preferred by older (compared to younger) workers and, therefore, facilitate positive work outcomes particularly in late career. Among others, Zacher et al. (2018) emphasized the importance of job autonomy, social embeddedness, and sense of meaning for aging workers. Therefore, allowing opportunities for higher job autonomy, task significance, skill variety, and social support may foster SAW across the lifespan (see also Cadiz, Rineer, \& Truxillo, 2019).

Both formal (e.g., Strategic Human Resource Management) and informal (e.g., i-deals between employees and their supervisors) human resource management (HRM) approaches are shown to be important for supporting workers' well-being and performance (Bal, 2015; Kooij, Jansen, Dikkers, \& De Lange, 2014). Some formal HRM practices (also referred to as "age-friendly," cf. Zacher et al., 2018) are viewed as having desirable effects especially for older workers. Kooij et al. (2014) conceptualized four bundles of HRM practices for aging workers, and among these are maintenance and development practices. Taneva and Arnold (2018) reported that a specific combination between some maintenance and development HRM practices predicted experiences of high work well-being (thriving at work) and high job performance in a large sample of older workers in the United Kingdom. These practices, most valued by the older workers, refer to access to training, recognition and respect, meaningful assignments, feedback from the supervisor, flexible working, and opportunities to work longer. In addition, there is considerable evidence about the value of informal work arrangements and the role of supervisors in late career. For example, Bal (2015) concluded that "individualization of career arrangements will be increasingly important in the sustainability of contemporary careers" (Bal, 2015, p. 1). Based on this evidence, we suggest that workers' access to a combination of certain HRM practices with informal work agreements (ideals) may be instrumental for achieving SAW in organizations.

\section{Outcomes of Successful Aging at Work}

Theoretically, SAW has been positively associated with various individual-level work outcomes, among which work motivation, job performance, health, and well-being (Zacher, 2015a). So far, only a few studies have provided empirical support for some of these associations. For example, Taneva and Arnold (2018) reported three pathways to SAW in organizations that demonstrate the role of personal (SOC) and organizational (HRM) strategies for achieving high job performance. Moreover, they found that employees' psychological well-being mediated the relationships of personal and organizational strategies with selfrated job performance (task proactivity, extra- and in-role performance). Therefore, workers' well-being is an important and, perhaps more direct (compared to job performance), outcome of SAW.

Thriving at work is described as "the joint sense of vitality and learning, which communicates a sense of progress or forward movement in one's self-development" (Spreitzer, Sutcliffe, Dutton, Sonenshein, \& Grant, 2005, p. 538). Experiences of thriving at work can be driven by both person and organizational factors (Spreizer, Porath, \& Gibson, 2012). Thriving at work is positively related to outcomes such as high job and career role performance, positive work behaviors, work-related, and overall well-being (Porath, Spreitzer, Gibson, \& Garnett, 2012). Two studies by Taneva and colleagues (Taneva et al., 2016; Taneva \& Arnold, 2018) demonstrated that high levels of thriving at work are also common among older workers. 
Hence, experiences of thriving at work can happen at all career stages. Moreover, thriving at work can be viewed as both a form of psychological well-being (cf. Spreitzer et al., 2005; Warr, 2007) and a criterion for SAW (Taneva \& Arnold, 2018).

As opposed to thriving, the emerging construct of surviving at work is described as the individual's tendency to preserve one's mental and physical resources by limiting one's work activities and perspectives to cope with work conditions that are perceived as highly demanding (Taneva et al., 2016). Such a preservation experience may indicate reduced levels or lack of SAW. However, Taneva and Arnold (2018) discovered that, at least in some circumstances, experiences of surviving at work may be indeed a sign of sustained person-environment fit and, ultimately, of SAW. This is also in line with Kooij's definition of SAW as sustainable maintenance (Kooij, 2015) and the definition of AAW by Zacker and colleagues (Zacher et al., 2018). Hence, surviving at work may be another indicator of SAW.

Furthermore, as outlined in the previous sections, individuals' experiences of SAW can be enhanced using personal (e.g., SOC) or organizational (e.g., HRM, i-deals, and job design) strategies that facilitate the regulation of one's resources. The concept of work ability (WA) describes the balance (fit) between work and personal resources (Tuomi, Ilmarinen, Jahkola, Katajarinne, \& Tulkki, 1998). Recent lifespan research has demonstrated that certain work (e.g., job control, feedback, and social support) and person (e.g., proactive behaviors) characteristics can support the maintenance and even the increase of WA, especially in late career (e.g., Weigl, Müller, Hornung, Zacher, \& Angerer, 2013). In this context, it has been suggested that WA may be a useful criterion for SAW in late career (and beyond) (Kooij, 2015; Weigl et al., 2013).

\section{CONCLUSION}

We developed and established the psychometric properties of the first single measure for SAW in the contemporary meaning of this construct (cf. Zacher, 2015a). We view SAW as a developmental process that may occur at all career stages and can be captured through individual-level outcomes such as improved WA and well-being. We operationalized the construct SAW through two clusters of employees' perceptions: (a) individuals' psychological experiences of age-related growth in the workplace (SAW), and (b) Strategies for achieving SAW. Experiences of growth may refer to various areas of perceived agerelated positive changes, among which are improved knowledge and experience, social skills, emotion regulation, and adaptability in work-related situations (cf. Taneva et al., 2016). Strategies (or facilitators) for SAW may include both employee (e.g., proactive self-regulation) and organizational (e.g., job design, formal HRM and informal work arrangements) behaviors (cf. Bal, 2015; Baltes \& Baltes, 1990; Kooij et al., 2014; Taneva et al., 2016; Truxillo et al., 2012).

Subsequent empirical analysis with data from three consecutive MTurk samples with overall 417 workers in organizational setting revealed that the employees' experiences of SAW largely overlapped with the employees' perceptions of individual-level facilitators of SAW (i.e., personal strategies). A potential explanation may be that SAW could be also described as a process of active self-regulation, driven by the individual's motivation to pursue person-environment fit (Kooij et al., 2019). Thus, the employees' perceptions of both positive age-related changes and proactive self-regulating behaviors (personal strategies) may reflect the construct SAW in the sense of perceived age-related growth (cf. Taneva et al., 2016).

Furthermore, we examined the construct and criterion-related validity of the new scale by exploring the associations of the SAW scale's total score with the scores of six established measures of personal (SOC) and organizational (job design, formal HRM) strategies, as well as subjective criteria for SAW (WA, thriving and surviving at work). The empirical analyses confirmed all anticipated relationships, apart from the association between the SAW score and the Surviving at Work score, which appeared to be lacking statistical significance. This may be because we suggested surviving at work as a tentative criterion for SAW. Although employees' experiences of surviving at work may indicate maintenance of resources and thus sustained work outcomes (e.g., well-being) (cf. Kooij, 2015; Taneva \& Arnold, 2018), the construct 
surviving at work does not imply perceived growth as it is the case with SAW in Zacher's definition (cf. Zacher, 2015a).

\section{Contributions to Theory and Practice}

This study contributed to theory development in several ways. First, it systemized and further conceptualized key domains of the emerging construct SAW by linking person and environmental variables. Thus, it responded to recent calls for integrating multilevel factors when examining this construct (cf. Zacher, 2015a; Zacher et al., 2018). Second, it focused on the synergies between these domains and gained new insights into how they operate together, as well as separately. Third, it translated into practical terms each domain and explored its construct and criterion-related validity with regard to other, well-known constructs and demographic variables. Thus, new information about the content of each domain was found and a new measurement instrument was developed. From a practitioner point of view, this instrument will help training and development professionals to capture workers' experiences and potential to age successfully in organizations. Moreover, this scale may help workers in monitoring and directing their own experiences of SAW. This, in turn, may inform organizational systems and initiatives aiming at supporting SAW and therefore ensure employees' higher work well-being and performance. In the longer term, supporting SAW from both individual and organizational perspectives may help in sustaining longer and healthier working lives.

\section{Limitations and Avenues for Future Research}

Future research needs to provide further evidence of the validity of the SAW scale. For example, we found a significant positive relationship of age with organizational tenure, but we did not find evidence of age-related differences regarding the employees' self-perceptions of SAW. The lack of evidence of agerelated differences may be because the age characteristics of our participant groups were not specified well enough or because generally there is no clear, definitive criterion for dividing workers into "older" and "younger" (cf. Zaniboni, Kmicinska, Truxillo, Kahn, Paladino, \& Fraccaroli, 2019). Also, our pilot and calibration samples did not include any participants of over 60 years of age. It may be the case that people aged 60 years and over, who stay in the workforce, experience SAW differently compared to workers at younger ages. Future research should address this issue. In addition, it is possible that various moderators play a role in workers' aging (cf. Felicitas, Goecke, \& Kunze, 2016; Zacher et al., 2018). Importantly, the lack of evidence of age-related differences in the employees' self-perceptions of SAW may indicate that the measure, which we suggest, captures SAW at all career stages (cf. Zacher et al., 2018).

Furthermore, at this stage of the scale's development we focused on several job characteristics and a small range of demographic variables. We did not collect information about those work characteristics, which may be negatively associated with SAW at some career stages (e.g., high physical demands in late career), the participants' occupations, organizations, and industrial sectors. Such information would help contextualizing and understanding better the SAW construct and measure. Also, we relied on data from MTurk workers, which may limit the generalizability of the study results (cf. Walters, Christakis, \& Wright, 2018). In the future, SAW researchers should seek to collect data from workers at all career stages and various occupational/ industrial/cultural settings with non-MTurk samples. This will allow comparisons between groups of workers at different career stages/occupations/sectors/cultures. To better understand the age dimension of SAW, investigators should consider other age constructs (e.g., subjective age) in addition to workers' chronological age (cf. Felicitas et al., 2016) and follow a longitudinal approach (cf. Kooij et al., 2019). In addition, in this study we captured the associations between SAW and only three work-related outcomes (i.e., WA and psychological well-being, understood as both thriving at work and surviving at work), all of which self-rated. Data regarding other work outcomes (e.g., workers' performance, health, other forms of well-being, job attitudes, work motivation, expectations, etc.; cf. Zacher, 2015a) and from multiple sources will be most valuable in future research with the SAW scale. 


\section{REFERENCES}

Abraham, J.D., \& Hansson, R.O. (1995). Successful aging at work: An applied study of selection, optimization, and compensation through impression management. Journals of Gerontology, Series B: Psychological Sciences and Social Sciences, 50, 94-103. doi:10.1093/geronb/50B.2.P94

Bal, P. (2015). Sustainable careers: enabling older workers to continue working through individualized work arrangements. In A. De Vos and B. Van der Heijden, (Eds.), Handbook of research on sustainable careers (pp. 304-318). Cheltenham, UK: Edward Elgar.

Baltes, P.B., \& Baltes, M.M. (1990). Psychological perspectives on successful aging: The model of selective optimization with compensation. In P. B. Baltes \& M. M. Baltes. (Eds.), Successful aging: Perspectives from the behavioral sciences (pp. 1-34). New York, NY: Cambridge University Press. doi:10.1017/CBO9780511665684.003

Boateng, G.O., Nielands, T.B., Frongillo, E.A., Melgar-Quinonez, H.R., \& Young, S.L. (2018). Best practices for developing and validating scales for health, social, and behavioral research: A primer. Frontiers of Public Health, 6, 149.doi:10.3389/fpubh.2018.00149

Cadiz, D.M., Rineer, J.R., \& Truxillo, D.M. (2019). Lifespan perspectives on job and work design. In B. B. Baltes, C. W. Rudolph, \& H. Zacher (Eds.), Work across the lifespan (pp. 263-290). London, UK: Academic Press, Elsevier.

Carstensen, L.L., Turan, B., Scheibe, S., Ram, N., Ersner-Hershfield, H., Samanez-Larkin, G.R., ... \& Nesselroade, J.R. (2011). Emotional experience improves with age: evidence based on over 10 years of experience sampling. Psychology and Aging, 26, 21-33. doi:10.1037/a0021285

Cattell, R.B. (1966). The scree test for the number of factors. Multivariate Behavioral Research, 1, 245276. doi:10.1207/s15327906mbr0102_10

Dinno, A., \& Dinno, M.A. (2018). Package "paran.” Dortmund, Germany: R package version 1.5.2

Felicitas, T., Goecke, E., \& Kunze, F. (2016). The moderation role of subjective age in the chronological age/absenteeism relationship in teams. Academy of Management Proceedings, (1), 12970. doi:10.1080/1359432X.2018.1485651

Freund, A.M., \& Baltes, P.B. (2002). Life-management strategies of selection, optimization and compensation: Measurement by self-report and construct validity. Journal of Personality and Social Psychology, 82(4), 642-662. doi:10.1037/0022-3514.82.4.642

Horn, J.L. (1965). A rationale and test for the number of factors in factor analysis. Psychometrika, 30 , 179-185. doi:10.1007/BF02289447

Kanfer, R., \& Ackerman, P.L. (2004). Aging, adult development, and work motivation. Academy of Management Review, 29(3), 440-458. doi:10.2307/20159053

Kim, D. (2017). The impact of learning management systems on academic performance: Virtual Competency and student Involvement. Journal of Higher Education Theory and Practice, 17(2). Retrieved from https://articlegateway.com/index.php/JHETP/article/view/1564

Kooij, D.T.A.M. (2015). Successful aging at work: The active role of employees. Work, Aging and Retirement, 1(4), 309-319. doi:10.1093/workar/wav018

Kooij, D.T.A.M., Jansen, P.G.W., Dikkers, J.S.E., \& De Lange, A.H. (2014). Managing aging workers: A mixed methods study on bundles of HR practices for aging workers. The International Journal of Human Resource Management, 25(15), 2192-2212. doi:10.1080/09585192.2013.872169

Kooij, D.T.A.M., Zacher, H., Wang, M., \& Heckhausen, J. (2019). Successful aging at work: A process model to guide future research and practice. Industrial and Organizational Psychology: Perspectives on Science and Practice. Retrieved on 13.08.2019 from ResearchGate.

Moghimi, D., Scheibe, S., \& Freund, A. (2019). The model of selection, optimization, and compensation. In B. B. Baltes, C. W. Rudolph, \& H. Zacher (Eds.), Work across the lifespan (pp. 81-110). London, UK: Academic Press, Elsevier. eBook ISBN: 9780128127575 
Moghimi, D., Zacher, H., Scheibe, S., \& Van Yperen, N.W. (2017). The selection, optimization, and compensation model in the work context: A systematic review and meta-analysis of two decades of research. Journal of Organizational Behavior, 38(2), 247-275. doi:10.1002/job.2108

Morgeson, F.P., \& Humphrey, S.E. (2006). The Work Design Questionnaire (WDQ): Developing and validating a comprehensive measure for assessing job design and the nature of work. Journal of Applied Psychology, 91, 1321-1339. doi:10.1037/0021-9010.91.6.1321

Olson, D., \& Shultz, K. (2019). Lifespan perspectives on successful aging at work. In B. B. Baltes, C. W. Rudolph, \& H. Zacher (Eds.), Work across the lifespan (pp. 215-234). London, UK: Academic Press, Elsevier. eBook ISBN: 9780128127575

Porath, C., Spreitzer, G., Gibson, C., \& Garnett, F.G. (2012). Thriving at work: Toward its measurement, construct validation, and theoretical refinement. Journal of Organizational Behavior, 33(2), 250 275. doi:10.1002/job.756

Revelle, W. (2019). psych: Procedures for personality and psychological research. Evanston, IL: Northwestern University, Evanston. R package version 1.8.12

Robson, S.M., \& Hansson, R.O. (2007). Strategic self-development for successful aging at work. International Journal of Aging and Human Development, 64(4), 331-359. doi:10.2190/Q303171U-7686-8517

Robson, S.M., Hansson, R.O., Abalos, A., \& Booth, M. (2006). Successful aging: Criteria for aging well in the workplace. Journal of Career Development, 33(2), 156-177. doi:10.1177/0894845306292533

Ruscio, J., \& Roche, B. (2012). Determining the number of factors to retain in an exploratory factor analysis using comparison data of a known factorial structure. Psychological Assessment, 24(2), 282-292. doi:10.1037/a0025697

Spreitzer, G.M., Porath, C.L., \& Gibson, C.B. (2012). Toward human sustainability: How to enable more thriving at work. Organizational Dynamics, 41(2), 155-162. doi:10.1016/j.orgdyn.2012.01.009

Spreitzer, G.M., Sutcliffe, K.M., Dutton, J., Sonenshein, S., \& Grant, A.M. (2005). A socially embedded model of thriving at work. Organization Science, 16(5), 537-549. doi:10.1287/orsc.1050.0153

Taneva, S.K., \& Arnold, J. (2018). Thriving, surviving and performing in late career: A mixed-method study of pathways to successful aging in organizations. Work, Aging and Retirement, 4(2), 189212. doi:10.1093/workar/wax027

Taneva, S.K., Arnold, J., \& Nicolson, R. (2016). The experience of being an older worker in an organization: a qualitative analysis. Work, Aging and Retirement, 2(4), 396-414. doi: 10.1093/workar/waw011

Truxillo, D.M., Cadiz, D.M., Rineer, J.R., Zaniboni, S., \& Fraccaroli, F. (2012). A lifespan perspective on job design: Fitting the job and the worker to promote job satisfaction, engagement, and performance. Organizational Psychology Review, 2(4), 340-360. doi: $10.1177 / 2041386612454043$

Tuomi, K., Ilmarinen, J., Jahkola, A., Katajarinne, L., \& Tulkki, A. (1998). Work Ability Index 2nd Edition. Helsinki: Institute of Occupational Health.

Velicer, W. (1976). Determining the number of components from the matrix of partial correlations. Psychometrika, 41, 321-327. doi:10.1007/BF02293557

Walters, K., Christakis, D.A., \& Wright, D.R. (2018). Are Mechanical Turk worker samples representative of health status and health behaviors in the U.S.? PloS one, 13(6), e0198835. doi:10.1371/journal.pone.0198835

Warr, P. (2001). Age and work behavior: Physical attributes, cognitive abilities, knowledge, personality traits and motives. In C. L. Cooper, \& I. T. Robertson (Eds.), International review of industrial and organizational psychology (pp. 1-36). New York, NY: Wiley.

Warr, P. (2007). Work, happiness, and unhappiness. Mahwah, NJ: Erlbaum. eBook ISBN 9780203936856. 
Weigl, M., Müller, A., Hornung, S., Zacher, H., \& Angerer, P. (2013). The moderating effects of job control and selection, optimization, and compensation strategies on the age-work ability relationship. Journal of Organizational Behavior, 34(5), 607-628. doi:10.1002/job.1810

Zacher, H. (2015a). Successful aging at work. Work, Aging and Retirement, 1(1), 4-25. doi: 10.1093/workar/way002

Zacher, H. (2015b). The importance of a precise definition, comprehensive model, and critical discussion of successful aging at work. Work, Aging and Retirement, 1(4), 320-333. doi:10.1093/workar/wav020

Zacher, H., Kooij, D. T.A.M., \& Beier, M.E. (2018). Active aging at work: Contributing factors and implications for organizations. Organizational Dynamics, 47(1), 37-45. doi:10.1016/j.orgdyn.2017.08.001

Zacher, H., \& Rudolph, W. (2017). Successful aging at work and beyond: A review and critical perspective. In S. Profili, A. Sammarra , L. Innocenti (Ed.) Age Diversity in the Workplace (Advanced Series in Management, Volume 17) (pp. 35 - 64). Bradford, UK: Emerald Publishing Limited. eISBN: 978-1-78743-073-0

Zaniboni, S., Kmicinska, M., Truxillo, D.M., Kahn, K., Paladino M.P., \& Fraccaroli, F. (2019). Will you still hire me when I am over 50? The effects of implicit and explicit age stereotyping on resume evaluations. European Journal of Work and Organizational Psychology, 28(4), 453-467. doi:10.1080/1359432X.2019.1600506

\section{APPENDIX}

TABLE 1

\section{DEMOGRAPHIC CHARACTERISTICS OF COLLECTED SAMPLES}

\begin{tabular}{|c|c|c|c|}
\hline \multirow{2}{*}{ Sample characteristies } & \multicolumn{3}{|c|}{ Samples } \\
\hline & Prepilet & Pilot & Calibration \\
\hline Number of administered items & 58 & 67 & 45 \\
\hline Initial sample size & 80 & 263 & $18 \overline{8}$ \\
\hline Sample size after cleaning & 68 & 223 & 126 \\
\hline Age & $58(S D-6.0)$ & $44.7(S D-11.5)$ & $46.1(S D-11.1)$ \\
\hline Percent female & $59 \%$ & $58 \%$ & $45 \%$ \\
\hline Percent White & $79 \%$ & $81 \%$ & $86 \%$ \\
\hline Tenure at current organization & $=$ & $8.3(S D-6.5)$ & $9.8(S D-8.0)$ \\
\hline
\end{tabular}

TABLE 2

VALIDATION SCALES

\begin{tabular}{|c|c|c|c|c|}
\hline Measure & Authers & Variables & No. Items & Rating scale \\
\hline Thriving at Work & $\begin{array}{l}\text { Porath, Spreitzer, } \\
\text { Gibson \& Garnett } \\
\text { (2012) }\end{array}$ & $\begin{array}{l}\text { Thriving at work (energy and } \\
\text { learning) }\end{array}$ & 10 & $\begin{array}{l}\text { 7-pt. strongly disagree to } \\
\text { strongly agree }\end{array}$ \\
\hline Surviving at Work & $\begin{array}{l}\text { Taneva \& Amold } \\
(2018)\end{array}$ & Surviving at work & 3 & $\begin{array}{l}\text { S-pt. strongly disagnee to } \\
\text { strongly agree }\end{array}$ \\
\hline $\begin{array}{l}\text { Age-Friendly HR } \\
\text { Practices }\end{array}$ & $\begin{array}{l}\text { Taneva \& Amold } \\
\text { (2018) }\end{array}$ & Age-friendly practices (set of eight) & 8 & $\begin{array}{l}\text { 5-pt. strongly disagnee to } \\
\text { strongly agree }\end{array}$ \\
\hline SOC Strategies & $\begin{array}{l}\text { Freund \& Baltes } \\
(2002)\end{array}$ & $\begin{array}{l}\text { Selection, optimization and } \\
\text { compensation strategies }\end{array}$ & 12 & 4-pt. a litrle to exactly \\
\hline Work Ability Index & Tuomi et al. (1998) & Work ability & 1 & $\begin{array}{l}\text { 10-pt. completely unable to } \\
\text { work to onerall capacity at its } \\
\text { best }\end{array}$ \\
\hline $\begin{array}{l}\text { The Work Design } \\
\text { Questionnaire } \\
\text { (subscales) }\end{array}$ & $\begin{array}{l}\text { Morgeson \& } \\
\text { Humphrey (2006) }\end{array}$ & $\begin{array}{l}\text { Autonomy, task significance, skill } \\
\text { variety, social support, feedback from } \\
\text { others }\end{array}$ & 14 & $\begin{array}{l}\text { 5-pt. strongly disagree to } \\
\text { strangly agree }\end{array}$ \\
\hline
\end{tabular}




\section{TABLE 3 \\ LIST OF DEVELOPED ITEMS}

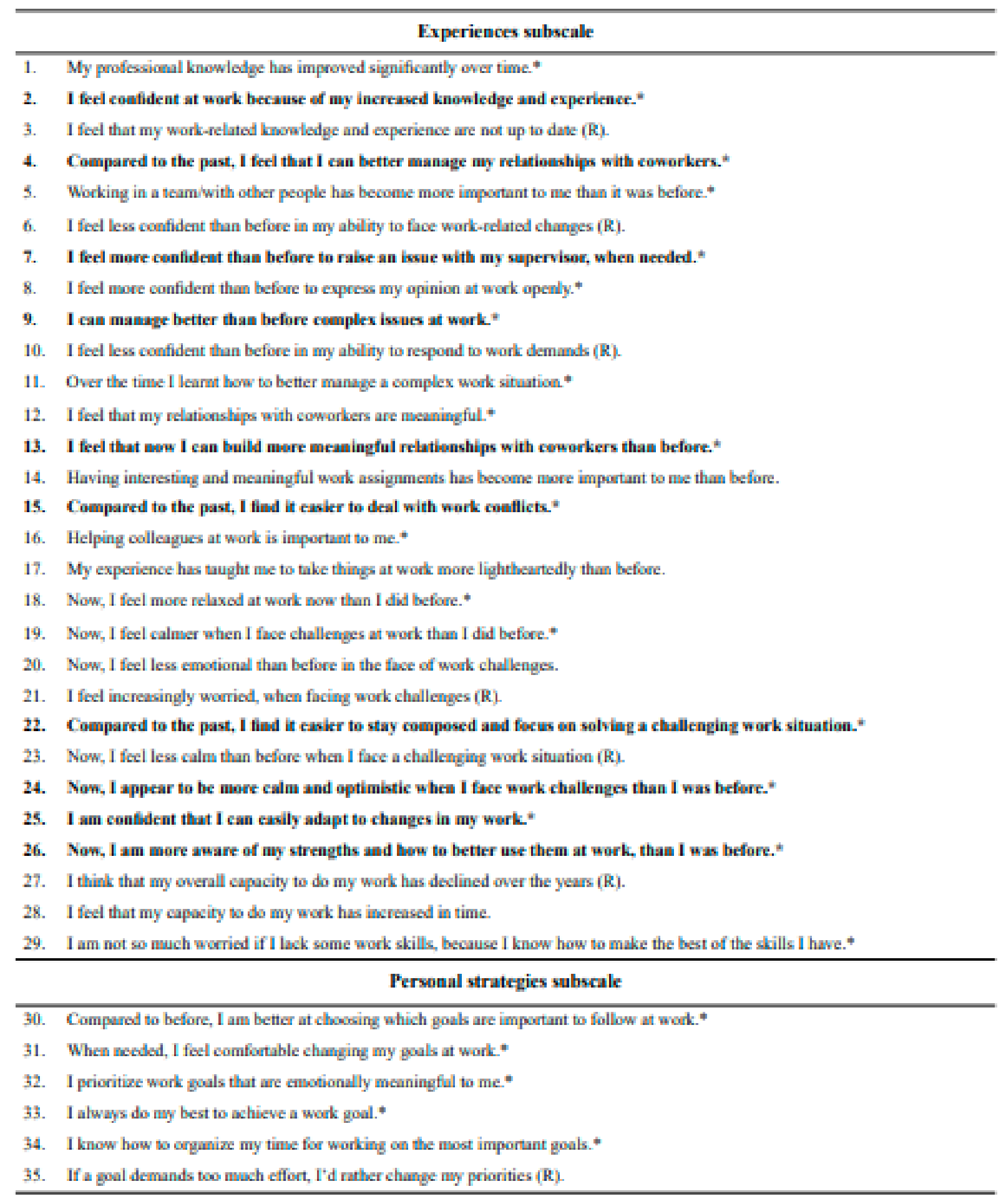




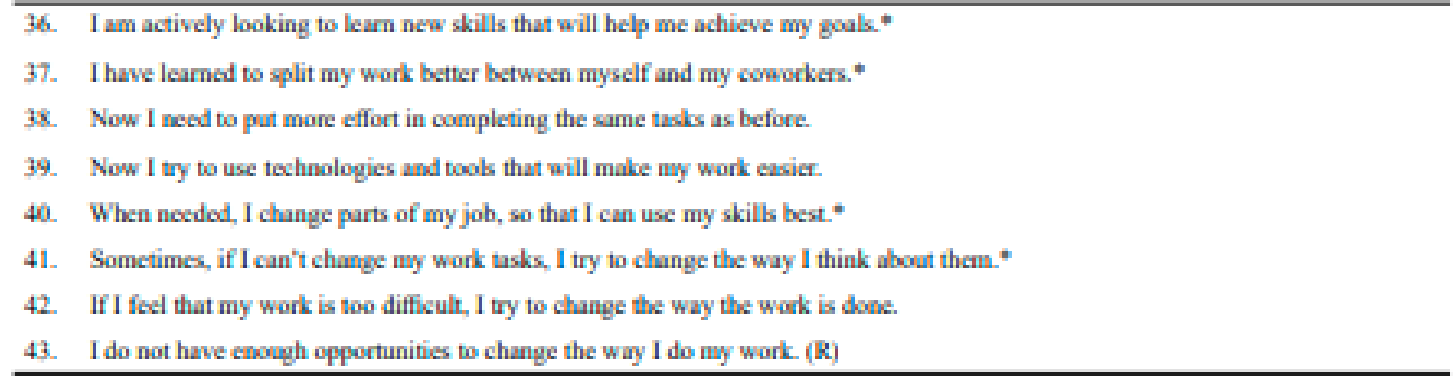

Organizational strategies subseale
44. When I need to, I can take additional or unpaid leave.
45. I have aecess to suitable training for updating my work skills.*
46. I feel that I don't have good enough opportunities for learning new skills at work (R).
47. My organization encourages me to never stop learning."
48. My organizatioe provides me with oppertanities to keep my work skills up to date-"
49. I feel recognized and respected at work:
50. I feel contident that I can stay with this orzanization for as long as I'd like."
51. I have a lot of flexibility at work.
52. I oflen work on challenging and meaningful tasks.*
53. My wodk has significance for society and the life of others.
54. My wodk involves leaming new things.*
55. My wodk does not imolve much variety (R).
5. I can always rely oe prompt and helpful feedhack from my supervisec."
57. My supervisor helps me to improve my work.
58. I am free to decide how to do my job.*
59. I don't have the freedom to do my work as I desire (R)
60. I have a great deal of autonomy at work.
61. My wodk involves meeting and getting wo know a lot of people.
62. I get support and understanding from my cowodkers.
63. I get support and understanding from my supervisor.*
64. My wodk team is one of the best things about my job."
65. My organization is open to nezotiatine personalized work arrangements with employees.
66. In my erganization, supervisors are flesible with the needs of their employees."
67. If necessary, I can negotiate with my supervisor special work arrangements that fit ny skills and eircumstanoes.

None. Items with asterisks $(*)$ included in the calibration sample, items in bold retained in the final version of the scale. 
TABLE 4

ITEM ANALYSES

\begin{tabular}{|c|c|c|c|c|c|c|c|c|c|c|c|c|c|c|c|c|c|c|c|}
\hline \multirow{3}{*}{ Item } & \multicolumn{2}{|c|}{ Pilot study } & \multicolumn{16}{|c|}{ Calibration study } & \multirow{3}{*}{ Alphas } \\
\hline & \multirow{2}{*}{ ICC } & \multirow{2}{*}{$\begin{array}{c}\text { Pilot } \\
\text { decision }\end{array}$} & \multirow{2}{*}{ ICC } & \multicolumn{5}{|c|}{ Loadings 1} & \multicolumn{3}{|c|}{ Laadings 2} & \multicolumn{2}{|c|}{ Loadings 3} & \multicolumn{2}{|c|}{ Loadings 4} & \multirow{2}{*}{$\begin{array}{c}\text { Final } \\
\text { decision }\end{array}$} & \multicolumn{2}{|c|}{$\begin{array}{c}\text { Final } \\
\text { loadings }\end{array}$} & \\
\hline & & & & Exp1 & Exp2 & PS & os1 & $\cos 2$ & Exp & PS & os & Exp & os & Exp & os/s & & Exp & os/s & \\
\hline item 1 & 54 & & .54 & .53 & .05 & & & & .57 & & & 55 & .07 & .48 & .13 & drop-1 & & & \multirow{29}{*}{ 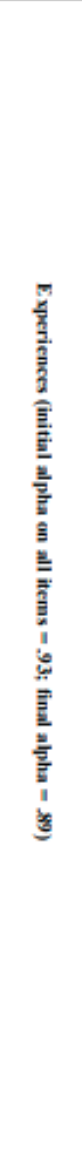 } \\
\hline item 2 & .62 & & .61 & .66 & -.02 & & & & .64 & & & 54 & .15 & .51 & .18 & keep-c & .54 & .16 & \\
\hline item 3 & .27 & drop & & & & & & & & & & & & & & & & & \\
\hline item 4 & .66 & & .69 & .41 & 39 & & & & .71 & & & .69 & .01 & .73 & .04 & kecp & .69 & .01 & \\
\hline item 5 & .40 & & .51 & .04 & .72 & & & & & & & & & & & & & & \\
\hline item 6 & .40 & drop & & & & & & & & & & & & & & & & & \\
\hline item 7 & .52 & & .59 & .60 & .03 & & & & .62 & & & .26 & .46 & .30 & .43 & keep-c & .33 & .39 & \\
\hline item 8 & .59 & & .60 & .61 & .03 & & & & .63 & & & .23 & 50 & .28 & .46 & drop-w & & & \\
\hline item 9 & .66 & & .65 & .73 & .04 & & & & .68 & & & .77 &. .10 & .74 & .08 & kecp & .75 & .07 & \\
\hline item 10 & .44 & drop & & & & & & & & & & & & & & & & & \\
\hline item 11 & .58 & & .57 & .60 & .00 & & & & .59 & & & .70 &. .10 & .64 & .06 & drop-r $-\mathrm{r}$ & & & \\
\hline item 12 & .54 & & .63 & .03 & .86 & & & & .62 & & & .49 & .18 & .53 & .15 & drop-r & & & \\
\hline item 13 & .65 & & .69 & .19 & .66 & & & & .68 & & & .54 & .17 & .61 & .10 & keep & .56 & .14 & \\
\hline item 14 & .42 & drop & & & & & & & & & & & & & & & & & \\
\hline item 15 & .68 & & .69 & .72 & .01 & & & & .72 & & & .59 & .12 & .66 & .07 & kecp & .68 & .07 & \\
\hline item 16 & .49 & & .51 & .05 & .60 & & & & .52 & & & .52 & .05 & .50 & .06 & drop-1 & & & \\
\hline item 17 & 53 & drop & & & & & & & & & & & & & & & & & \\
\hline item 18 & .69 & & .71 & .73 & .04 & & & & .75 & & & .57 & .19 & .64 & .13 & drop-r & & & \\
\hline item 19 & .76 & & .74 & .77 & .03 & & & & .78 & & & .63 & .15 & .70 & .10 & drop- $r$ & & & \\
\hline item 20 & 57 & drop & & & & & & & & & & & & & & & & & \\
\hline item 21 & .46 & drop & & & & & & & & & & & & & & & & & \\
\hline item 22 & .61 & & .66 & .65 & .06 & & & & .69 & & & .68 & -.01 & .74 & .06 & kecp & .73 &. .04 & \\
\hline item 23 & .48 & drop & & & & & & & & & & & & & & & & & \\
\hline item 24 & .73 & & .74 & .66 & .15 & & & & .77 & & & .75 & .01 & .81 & .05 & kecp & .76 & .01 & \\
\hline item 25 & .64 & & .58 & .66 & .04 & & & & .61 & & & .54 & .12 & .52 & .13 & keep-c & .55 & .11 & \\
\hline item 26 & .62 & & .60 & .73 &. .11 & & & & .62 & & & .77 &. .14 & .69 & .09 & kecp & .67 & .08 & \\
\hline item 27 & .32 & drop & & & & & & & & & & & & & & & & & \\
\hline item 28 & .56 & drop & & & & & & & & & & & & & & & & & \\
\hline item 29 & .41 & & .30 & .47 &. .18 & & & & & & & & & & & & & & \\
\hline
\end{tabular}




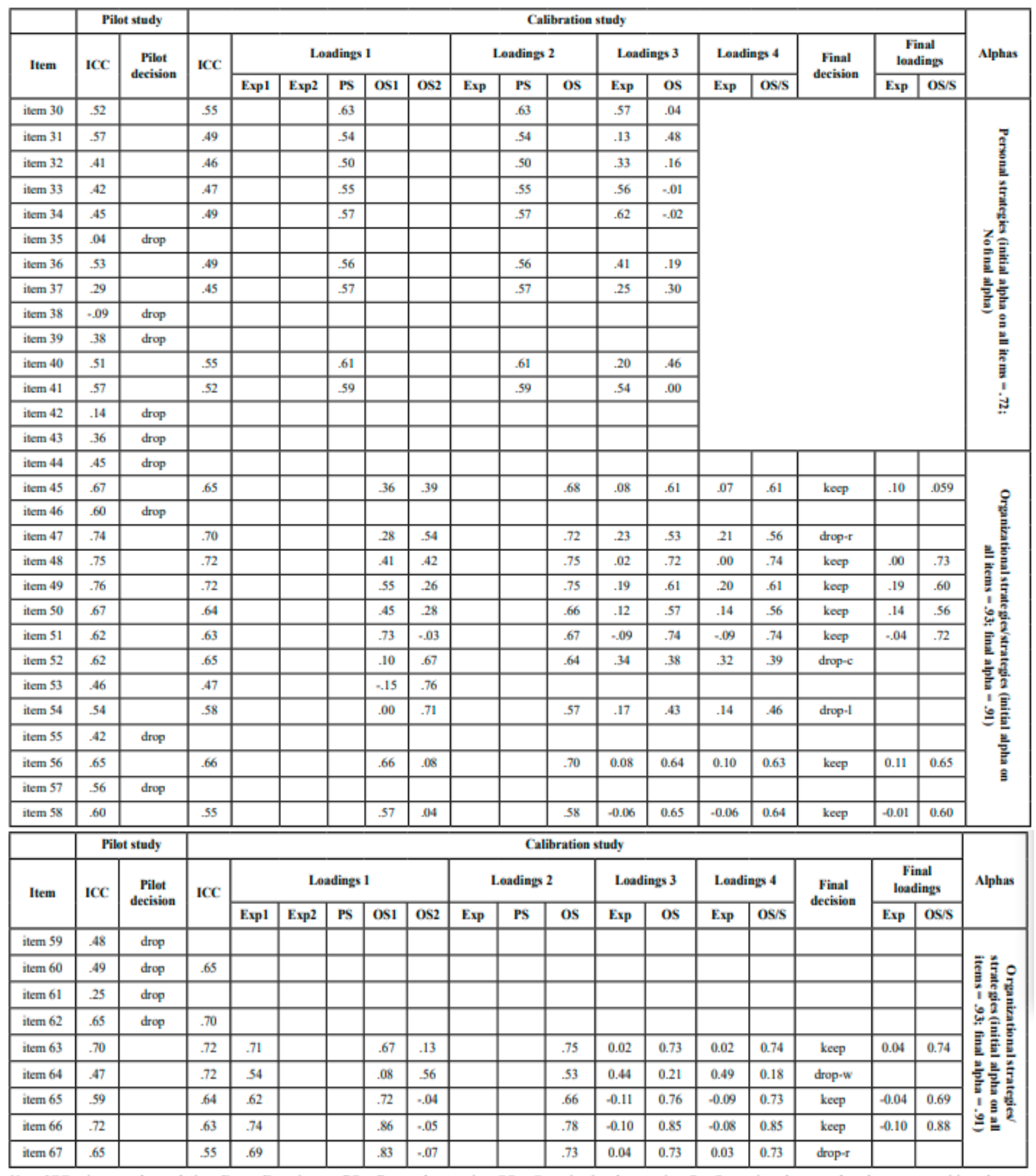

Note. ICC = item-total correlation; Exp = Experiences; $\mathrm{PS}=$ Personal strategies; $\mathrm{OS}=$ Organizational strategies; $\mathrm{S}=\mathrm{Strategies} ;$ drop- $\mathrm{r}=\mathrm{drop}$ because repetitive; drop-w $=$ drop because loads on the wrong factor, drop-l $=$ drop because low loading(s); drop- $\mathrm{c}=$ drop because cross-loading; keep $=$ keep; keep- $\mathrm{c}=\mathrm{keep}$ for construct-coverage reasons. 
TABLE 5

DESCRIPTIVE STATISTICS AND INTER-CORRELATIONS WITH VALIDATION SCALES

\begin{tabular}{|c|c|c|c|c|c|c|c|c|c|c|c|c|c|c|c|c|c|c|c|}
\hline Variable & 1. & 2. & 3. & 4. & 5. & 6. & 7. & 8. & 9. & 10. & 11. & 12. & 13. & 14. & 15. & 16. & 17. & 18. & 19. \\
\hline 1.Total score & .93 & & & & & & & & & & & & & & & & & & \\
\hline 2. Experiences & $.89 * *$ & .89 & & & & & & & & & & & & & & & & & \\
\hline 3. Strategies & $.94 * *$ & $.67 * *$ & .91 & & & & & & & & & & & & & & & & \\
\hline 4. Thriving at work & $.72 * *$ & $.66^{* *}$ & $.65 * *$ & .93 & & & & & & & & & & & & & & & \\
\hline 5. Surviving at work &. .12 & .07 & -.14 & $-.27 * *$ & .73 & & & & & & & & & & & & & & \\
\hline 6. HRM practices & $.74 * *$ & $52 * *$ & $.80 * *$ & $.69 * *$ & $-.23^{*}$ & .88 & & & & & & & & & & & & & \\
\hline 7. SOC elective selection & $.35 * *$ & $42 * *$ & $.25 * 0$ & $.32 * *$ & $.22^{*}$ & $.23 * *$ & .73 & & & & & & & & & & & & \\
\hline 8. SOC loss-based selection & $.22^{*}$ & $.32 * *$ & .12 & $.22^{*}$ & .12 & .17 & $.53 * *$ & .61 & & & & & & & & & & & \\
\hline 9. SOC compensation & $.46^{* *}$ & $.54 * *$ & $.33 * *$ & $.50^{* * *}$ & -.10 & $.40 * *$ & $.51 * *$ & $.38 * *$ & .86 & & & & & & & & & & \\
\hline 10. SOC optimization & $42 * *$ & $.47 * *$ & $32 * *$ & $.44 * *$ & -.07 & $34 * *$ & $.42 * *$ & $.46^{* *}$ & $.58 * *$ & .59 & & & & & & & & & \\
\hline 11. SOC (total) & $.47^{* *}$ & $.56^{* *}$ & $33 * *$ & $.47^{* * *}$ & .06 & $36 * *$ & $.80 * *$ & $.76^{* *}$ & $.78 * *$ & $.78 * *$ & .85 & & & & & & & & \\
\hline 12. Work ability & $.30 * *$ & $.23 * *$ & $31 * *$ & $.41^{* *}$ & $-.30^{* *}$ & $22^{*}$ & .00 & -.03 & $.25 * *$ & $.23 * *$ & .14 & - & & & & & & & \\
\hline 13. Autonomy & $.74 * *$ & $.54^{* *}$ & $.78 * *$ & $.56 * *$ & -.07 & $.68 * *$ & $22^{*}$ & .12 & $.30^{* *}$ & $.26 * *$ & $.29 * *$ & $31 * *$ & .85 & & & & & & \\
\hline 14. Task significance & $.50 * 4$ & $.37 * *$ & $.52 * *$ & $.41^{* *}$ & -.11 & $.55 * *$ & .11 & .09 & $.30^{* *}$ & $.31 * *$ & $.25 * *$ & .06 & $.47^{* * *}$ & .80 & & & & & \\
\hline 15. Skill variety & $.58 * *$ & $.46^{* *}$ & $59 * *$ & $.49 * *$ & -.10 & $s 1^{* *}$ & .12 & -.01 & $.22 *$ & $.24 * *$ & $.18^{*}$ & $.24 * *$ & $.70 * *$ & $.47 * *$ & .84 & & & & \\
\hline 16. Social support & $.77^{* * *}$ & $.65 * *$ & $.74 * *$ & $.52 * *$ & -.17 & $.69 * *$ & $.24 * *$ & .11 & $.31 * *$ & $.35 * *$ & $.32^{* *}$ & $.22^{*}$ & $.58 * *$ & $.52^{* *}$ & $.52^{* * *}$ & .71 & & & \\
\hline 17. Feedhack from others & $.76 * *$ & $.58 * *$ & $.78 * *$ & $.59 * *$ & $-.20^{*}$ & $.74 * *$ & $.28 * *$ & .17 & $.32^{* * *}$ & $.32^{* *}$ & $.35 * *$ & $.19^{*}$ & $.64^{* *}$ & $.51^{* *}$ & $.45^{* *}$ & $.72^{* * *}$ & .85 & & \\
\hline 18. Age & .07 & .09 & 0.04 & .07 & .02 & .09 & .06 & .13 & .07 & .11 & .12 & -.02 & -.00 & .09 & .07 & .03 & .07 & - & \\
\hline 19. Organizational tenure & .06 & .05 & .07 & -.03 & .16 & .04 & .06 & .12 & .09 & .08 & .12 & -10 & .00 & .06 & .06 & -.05 & .02 & $.43^{* *}$ & - \\
\hline Mean & 4.00 & 3.95 & 3.74 & 5.30 & 3.15 & 3.43 & 2.57 & 2.43 & 3.13 & 2.76 & 2.72 & 8.33 & 3.73 & 3.52 & 4.03 & 3.65 & 3.61 & 45.2 & 8.85 \\
\hline SD & .63 & .61 & .78 & 1.20 & .95 & .86 & .68 & .66 & .64 & .61 & 51 & 1.33 & .92 & 1.08 & .89 & .84 & 1.02 & 11.35 & 7.11 \\
\hline
\end{tabular}

\section{TRANSLATED VERSION: SPANISH}

Below is a rough translation of the insights presented above. This was done to give a general understanding of the ideas presented in the paper. Please excuse any grammatical mistakes and do not hold the original authors responsible for these mistakes.

\section{VERSION TRADUCIDA: ESPAÑOL}

A continuación se muestra una traducción aproximada de las ideas presentadas anteriormente. Esto se hizo para dar una comprensión general de las ideas presentadas en el documento. Por favor, disculpe cualquier error gramatical y no responsabilite a los autores originales de estos errores.

\section{INTRODUCCIÓN}

El concepto emergente de envejecimiento exitoso en el trabajo (SAW) tiene sus raíces en las interpretaciones contemporáneas de las teorías del desarrollo organizacional y de la vida útil. Enfatiza el importante papel de los lugares de trabajo para el envejecimiento exitoso (Olson y Schultz, 2019), lo que implica que las experiencias largas y positivas relacionadas con el trabajo pueden sostenerse a través de una adaptación exitosa a los cambios intrainteulares (por ejemplo, relacionados con la edad) y externos (por ejemplo, ambientales) (Kooij, 2015; Olson \& Schultz, 2019; Zacher, 2015a). Kooij (2015) describió SAW como el mantenimiento sostenible de la capacidad de trabajo, la salud y la motivación de un individuo a lo largo del ciclo de vida laboral. En esta definición, el papel activo del empleado para la creación de recursos de trabajo y asegurar un ajuste continuo persona-entorno es de importancia clave. Zacher (2015a, b) señaló que SAW no se limita a mantener los niveles actuales de funcionamiento y puede referirse al desarrollo o crecimiento de un trabajador. Por lo tanto, saw puede verse como una trayectoria intra-individual relacionada con la edad hacia un resultado laboral (por ejemplo, bienestar o desempeño laboral) que se desvía positivamente de la trayectoria promedio relacionada con la edad. Incluso un empleado que ha 
experimentado una disminución en un resultado laboral a lo largo del tiempo puede estar envejeciendo con éxito en el trabajo cuando su trayectoria es más positiva que la trayectoria promedio relacionada con la edad (Zacher, 2015a, b).

De manera sistemática, Zacher, Kooij y Beier (2018) describieron los factores que contribuyen al envejecimiento activo en el trabajo (AAW), que es una construcción que se superpone de alguna manera con SAW (cf. Zacher, 2015a). AAW se describe como la capacidad de los trabajadores para "mantener o mejorar (1) su bienestar físico, mental y social; (2) continuar mostrando altos niveles de compromiso y desempeño en el trabajo; y (3) experimentar un trato justo y seguridad en el empleo" en el proceso de envejecimiento (Zacher et al., 2018, p. 37). Debido a que el desarrollo es un proceso de por vida, AAW se refiere a trabajadores de todas las edades y etapas profesionales. Los factores multinivel (individuales, de equipo, organizacionales y de la sociedad en general) contribuyen a AAW / SAW (ver también Kooij, Zacher, Wang y Heckhausen, 2019). La idea de comprender AAW / SAW en relación con los factores internos (por ejemplo, persona) y externos (por ejemplo, ambientales) está en línea con la crítica continua de que al centrarse principalmente en el proceso de desarrollo intra-individual, es probable que las teorías de la esperanza de vida en general descuiden los efectos del contexto en el desarrollo del individuo (Olson y Schultz, 2019).

Dado el creciente interés en AAW / SAW desde una perspectiva de vida útil contemporánea (Zacher et al., 2018), es sorprendente que aún no se haya desarrollado una medida de este constructo. En dos estudios consecutivos en 2006 y 2007, Robson y sus colegas conceptualizaron criterios tentativos para el envejecimiento exitoso en el lugar de trabajo y desarrollaron un inventario para medirlos (Robson y Hansson, 2007; Robson, Hansson, Abalos, \& Booth, 2006). Zacher y Rudolph (2017) criticaron este modelo por descuidar los factores contextuales y los resultados del trabajo al centrarse completamente en los factores de la persona (estrategias de comportamiento). Además, Zacher \& Rudolph (2017) concluyeron que debido a las estrategias conductuales sugeridas por Robson et al. (Robson \& Hansson, 2007; Robson et al., 2006) no interactúan con la edad, estas estrategias no pueden considerarse estrategias de envejecimiento exitosas. Más recientemente, Kooij y sus colegas reconocieron la dificultad de operacionalizar y, por lo tanto, medir el constructo SAW (Kooij et al., 2019). Al igual que con Zacher (2015a), entendemos saw como un proceso de crecimiento intra-individual multidimensional, que está relacionado con la edad, asociado con diversos criterios subjetivos y objetivos, y puede ser facilitado (o limitado) por factores multinivel. Basado en la definición de SAW de Zacher (2015a) y el marco de factores AAW por Zacher et al. (2018), el propósito de nuestro estudio es ampliar el conocimiento de SAW mediante: (a) la operacionalización de facetas importantes del constructo SAW y (b) la creación de una sola medida de SAW. Nos centramos en las experiencias y resultados individuales de SAW, así como en los antecedentes personales y ambientales de SAW en el contexto organizacional. También consideramos las posibles interacciones de estos factores con la edad de los trabajadores (cf. Zacher et al., 2015a, 2018). Probamos las propiedades psicométricas de la nueva medida en tres muestras consecutivas de MTurk y sugerimos direcciones para futuras investigaciones.

\section{Operacionalización del Envejecimiento Exitoso en el Trabajo \\ Experiencias de los Empleados de SAW}

Los impactos de los cambios relacionados con la edad en los comportamientos y resultados laborales (especialmente en la carrera tardía) están ampliamente documentados. La naturaleza y el alcance de los cambios relacionados con la edad pueden variar significativamente entre los individuos debido a las diferencias en la persona y los factores ambientales. Es probable que estos cambios (por ejemplo, en las capacidades cognitivas, la personalidad y la motivación de los trabajadores) reflejen varias pérdidas y ganancias en el desarrollo a lo largo del ciclo de vida laboral (Kanfer y Ackerman, 2004; Warr, 2001). Recientemente, el número de estudios centrados en los aspectos positivos (es decir, ganancias del desarrollo) en lugar de los aspectos relacionados con la pérdida (es decir, disminuciones del desarrollo) del proceso de envejecimiento ha aumentado. Por ejemplo, en un estudio cualitativo con 37 trabajadores mayores (de 55 años o más) de dos países (Bulgaria y Reino Unido) y dos sectores industriales (salud y tecnologías de la información y la comunicación), Taneva, Arnold y Nicolson (2016) encontraron que los 
trabajadores de carrera tardía son muy conscientes de cómo los cambios relacionados con la edad pueden afectar su vida laboral y sus resultados. Es importante destacar que los trabajadores asocian algunos de estos cambios (por ejemplo, mayor conocimiento y experiencia, mayor experiencia social y emocional) con su desarrollo personal y profesional y, en última instancia, con mejores resultados laborales. Por lo tanto, es probable que las percepciones de los trabajadores sobre los cambios positivos relacionados con la edad (ganancias en el desarrollo; cf. Kanfer y Ackerman, 2004) indiquen las experiencias de los trabajadores con SAW.

En el estudio de Taneva et al. (2016), junto con sus percepciones de un mayor conocimiento y experiencia, los trabajadores (mayores) comunicaron percepciones de otras áreas de crecimiento personal y profesional, entre las que se encuentran el aumento de la autoconciencia y la confianza, la mejora de las habilidades sociales, la regulación de las emociones, la capacidad de resolver problemas complejos y la adaptación general a los cambios contextuales. Estos hallazgos, extraídos de las narrativas de los trabajadores, se corresponden con los resultados de investigaciones anteriores en la tradición de la esperanza de vida (por ejemplo, Carstensen et al., 2011; Kanfer \& Ackerman, 2004). Sugerimos que las conceptualizaciones de los trabajadores de los cambios intra-individuales positivos pueden dar forma a un dominio importante del constructo SAW.

\section{Estrategias Personales y Organizativas Para SAW}

Desde la perspectiva de la teoría de la esperanza de vida, el envejecimiento es un proceso proactivo de adaptación, impulsado por la autorregulación en forma de estrategias de gestión de la vida que los individuos aplican para hacer frente a los cambios relacionados con la edad (por ejemplo, pérdida, ganancia y / o reorganización de recursos) en sí mismos, así como los cambios en su entorno (Kanfer y Ackerman, 2004). El modelo de optimización selectiva con compensación (SOC) (Baltes \& Baltes, 1990) propone que es probable que los individuos utilicen estrategias que se dividen en tres categorías de procesos de autorregulación (selección, optimización y compensación) en sus intentos de adaptarse tanto a los cambios relacionados con la edad como a los cambios en su entorno. Estos comportamientos proactivos se vuelven más importantes con el aumento de la edad y pueden ser particularmente útiles en el lugar de trabajo. Comenzando con el trabajo pionero de Abraham y Hansson (1995), una gran cantidad de estudios ha demostrado los efectos positivos de las estrategias SOC en el bienestar y el rendimiento de los trabajadores (mayores) (por ejemplo, Moghimi, Scheibe y Freund, 2019; Moghimi, Zacher, Shreible y Van Yperen, 2017). Por lo tanto, las estrategias SOC pueden ser un mecanismo eficaz para lograr saw.

Combinando las ventajas del diseño del trabajo y las teorías de la vida útil, Truxillo, Cádiz, Rineer, Zaniboni y Fraccaroli, (2012) propusieron posibles efectos relacionados con la edad de tres subgrupos de características del trabajo (tarea, conocimiento y social) sobre el compromiso laboral, la satisfacción laboral y el desempeño laboral. Por ejemplo, sugirieron que debido a los cambios intra-individuales relacionados con la edad, algunas características del trabajo, como la autonomía laboral, la importancia de la tarea, la variedad de habilidades y el apoyo social, pueden ser preferidas por los trabajadores mayores (en comparación con los más jóvenes) y, por lo tanto, facilitar resultados laborales positivos, particularmente en la carrera tardía. Entre otros, Zacher et al. (2018) enfatizaron la importancia de la autonomía laboral, la integración social y el sentido de significado para los trabajadores que envejecen. Por lo tanto, permitir oportunidades para una mayor autonomía laboral, importancia de la tarea, variedad de habilidades y apoyo social puede fomentar el SAW a lo largo de la vida (ver también Cádiz, Rineer y Truxillo, 2019).

Se ha demostrado que los enfoques formales (por ejemplo, Gestión Estratégica de Recursos Humanos) e informales (por ejemplo, acuerdos i entre empleados y sus supervisores) son importantes para apoyar el bienestar y el rendimiento de los trabajadores (Bal, 2015; Kooij, Jansen, Dikkers y De Lange, 2014). Se considera que algunas prácticas formales de gestión de recursos humanos (también conocidas como "amigables con las personas mayores", cf. Zacher et al., 2018) tienen efectos deseables, especialmente para los trabajadores mayores. Kooij et al. (2014) conceptualizaron cuatro paquetes de prácticas de gestión de recursos humanos para trabajadores de edad avanzada, y entre estos se encuentran las prácticas de mantenimiento y desarrollo. Taneva y Arnold (2018) informaron que una combinación específica entre algunas prácticas de gestión de recursos humanos de mantenimiento y desarrollo predijo experiencias de 
alto bienestar laboral (prosperar en el trabajo) y alto rendimiento laboral en una gran muestra de trabajadores mayores en el Reino Unido. Estas prácticas, más valoradas por los trabajadores mayores, se refieren al acceso a la capacitación, el reconocimiento y el respeto, las asignaciones significativas, la retroalimentación del supervisor, el trabajo flexible y las oportunidades de trabajar más tiempo. Además, hay pruebas considerables sobre el valor de los acuerdos de trabajo informales y el papel de los supervisores en la carrera tardía. Por ejemplo, Bal (2015) concluyó que "la individualización de los arreglos de carrera será cada vez más importante en la sostenibilidad de las carreras contemporáneas" (Bal, 2015, p. 1). Sobre la base de esta evidencia, sugerimos que el acceso de los trabajadores a una combinación de ciertas prácticas de gestión de recursos humanos con acuerdos de trabajo informales (i-deals) puede ser fundamental para lograr saw en las organizaciones.

\section{Resultados del Envejecimiento Exitoso en el Trabajo}

Teóricamente, SAW se ha asociado positivamente con varios resultados laborales a nivel individual, entre los que se encuentran la motivación laboral, el rendimiento laboral, la salud y el bienestar (Zacher, 2015a). Hasta ahora, solo unos pocos estudios han proporcionado apoyo empírico para algunas de estas asociaciones. Por ejemplo, Taneva y Arnold (2018) informaron tres vías hacia SAW en organizaciones que demuestran el papel de las estrategias personales (SOC) y organizacionales (HRM) para lograr un alto rendimiento laboral. Además, encontraron que el bienestar psicológico de los empleados mediaba las relaciones de las estrategias personales y organizacionales con el desempeño laboral autoevaluado (proactividad de tareas, desempeño extra y en el rol). Por lo tanto, el bienestar de los trabajadores es un resultado importante y, quizás más directo (en comparación con el desempeño laboral), de SAW.

Prosperar en el trabajo se describe como "el sentido conjunto de vitalidad y aprendizaje, que comunica un sentido de progreso o movimiento hacia adelante en el autodesarrollo" (Spreitzer, Sutcliffe, Dutton, Sonenshein y Grant, 2005, p. 538). Las experiencias de prosperidad en el trabajo pueden ser impulsadas tanto por factores personales como organizacionales (Spreizer, Porath y Gibson, 2012). Prosperar en el trabajo se relaciona positivamente con resultados como un alto desempeño laboral y profesional, comportamientos laborales positivos, relacionados con el trabajo y bienestar general (Porath, Spreitzer, Gibson y Garnett, 2012). Dos estudios realizados por Taneva y sus colegas (Taneva et al., 2016; Taneva y Arnold, 2018) demostraron que los altos niveles de prosperidad en el trabajo también son comunes entre los trabajadores mayores. Por lo tanto, las experiencias de prosperar en el trabajo pueden ocurrir en todas las etapas de la carrera. Además, prosperar en el trabajo puede ser visto como una forma de bienestar psicológico (cf. Spreitzer et al., 2005; Warr, 2007) y un criterio para SAW (Taneva \& Arnold, 2018).

En lugar de prosperar, la construcción emergente de sobrevivir en el trabajo se describe como la tendencia del individuo a preservar los recursos mentales y físicos de uno al limitar las actividades y perspectivas de trabajo para hacer frente a las condiciones de trabajo que se perciben como altamente exigentes (Taneva et al., 2016). Tal experiencia de preservación puede indicar niveles reducidos o falta de SAW. Sin embargo, Taneva y Arnold (2018) descubrieron que, al menos en algunas circunstancias, las experiencias de supervivencia en el trabajo pueden ser de hecho un signo de ajuste sostenido personaentorno y, en última instancia, de SAW. Esto también está en línea con la definición de KOoij de SAW como mantenimiento sostenible (Kooij, 2015) y la definición de AAW de Zacker y sus colegas (Zacher et al., 2018). Por lo tanto, sobrevivir en el trabajo puede ser otro indicador de SAW.

Además, como se describió en las secciones anteriores, las experiencias de las personas con SAW se pueden mejorar utilizando estrategias personales (por ejemplo, SOC) u organizacionales (por ejemplo, HRM, i-deals y diseño de trabajos) que faciliten la regulación de los recursos. El concepto de capacidad de trabajo (WA) describe el equilibrio (ajuste) entre el trabajo y los recursos personales (Tuomi, Ilmarinen, Jahkola, Katajarinne y Tulkki, 1998). Investigaciones recientes sobre la esperanza de vida han demostrado que ciertas características de trabajo (por ejemplo, control del trabajo, retroalimentación y apoyo social) y de la persona (por ejemplo, comportamientos proactivos) pueden apoyar el mantenimiento e incluso el aumento de WA, especialmente en la carrera tardía (por ejemplo, Weigl, Müller, Hornung, Zacher y Angerer, 2013). En este contexto, se ha sugerido que WA puede ser un criterio útil para SAW en la carrera tardía (y más allá) (Kooij, 2015; Weigl et al., 2013). 


\section{CONCLUSIÓN}

Desarrollamos y establecimos las propiedades psicométricas de la primera medida única para SAW en el significado contemporáneo de este constructo (cf. Zacher, 2015a). Vemos SAW como un proceso de desarrollo que puede ocurrir en todas las etapas de la carrera y puede ser capturado a través de resultados a nivel individual, como la mejora de wa y el bienestar. Operacionalizamos el constructo SAW a través de dos grupos de percepciones de los empleados: (a) las experiencias psicológicas de los individuos de crecimiento relacionado con la edad en el lugar de trabajo (SAW), y (b) Estrategias para lograr SAW. Las experiencias de crecimiento pueden referirse a diversas áreas de cambios positivos percibidos relacionados con la edad, entre los que se encuentran la mejora del conocimiento y la experiencia, las habilidades sociales, la regulación de las emociones y la adaptabilidad en situaciones relacionadas con el trabajo (cf. Taneva et al., 2016). Las estrategias (o facilitadores) para SAW pueden incluir comportamientos tanto de los empleados (por ejemplo, autorregulación proactiva) como organizacionales (por ejemplo, diseño de trabajo, gestión formal de recursos humanos y arreglos de trabajo informales) (cf. Bal, 2015; Baltes \& Baltes, 1990; Kooij et al., 2014; Taneva et al., 2016; Truxillo et al., 2012).

El análisis empírico posterior con datos de tres muestras consecutivas de MTurk con un total de 417 trabajadores en el entorno organizacional reveló que las experiencias de saw de los empleados se superponían en gran medida con las percepciones de los empleados de los facilitadores a nivel individual de SAW (es decir, estrategias personales). Una posible explicación puede ser que la SAW también podría describirse como un proceso de autorregulación activa, impulsado por la motivación del individuo para buscar el ajuste persona-entorno (Kooij et al., 2019). Por lo tanto, las percepciones de los empleados tanto de los cambios positivos relacionados con la edad como de los comportamientos proactivos de autorregulación (estrategias personales) pueden reflejar el constructo SAW en el sentido de crecimiento percibido relacionado con la edad (cf. Taneva et al., 2016).

Además, examinamos la validez de constructo y relacionada con los criterios de la nueva escala explorando las asociaciones de la puntuación total de la escala SAW con las puntuaciones de seis medidas establecidas de estrategias personales (SOC) y organizativas (diseño de trabajo, HRM formal), así como criterios subjetivos para SAW (WA, prosperar y sobrevivir en el trabajo). Los análisis empíricos confirmaron todas las relaciones anticipadas, excepto la asociación entre la puntuación SAW y la puntuación Surviving at Work, que parecía carecer de significación estadística. Esto puede deberse a que sugerimos sobrevivir en el trabajo como un criterio tentativo para SAW. Aunque las experiencias de supervivencia de los empleados en el trabajo pueden indicar el mantenimiento de los recursos y, por lo tanto, los resultados de trabajo sostenidos (por ejemplo, el bienestar) (cf. Kooij, 2015; Taneva \& Arnold, 2018), el constructo que sobrevive en el trabajo no implica crecimiento percibido como es el caso de SAW en la definición de Zacher (cf. Zacher, 2015a).

\section{Contribuciones a La Teoría y La Práctica}

Este estudio contribuyó al desarrollo de la teoría de varias maneras. En primer lugar, sistematizó y conceptualizó aún más los dominios clave del constructo emergente SAW mediante la vinculación de variables personales y ambientales. Por lo tanto, respondió a los recientes llamamientos para integrar factores multinivel al examinar este constructo (cf. Zacher, 2015a; Zacher et al., 2018). En segundo lugar, se centró en las sinergias entre estos dominios y obtuvo nuevos conocimientos sobre cómo funcionan juntos, así como por separado. En tercer lugar, tradujo en términos prácticos cada dominio y exploró su constructo y validez relacionada con criterios con respecto a otros constructos y variables demográficas bien conocidos. Así, se encontró nueva información sobre el contenido de cada dominio y se desarrolló un nuevo instrumento de medición. Desde el punto de vista de los profesionales, este instrumento ayudará a los profesionales de la formación y el desarrollo a capturar las experiencias de los trabajadores y el potencial para envejecer con éxito en las organizaciones. Además, esta escala puede ayudar a los trabajadores a monitorear y dirigir sus propias experiencias de SAW. Esto, a su vez, puede informar los sistemas e iniciativas organizacionales destinados a apoyar a SAW y, por lo tanto, garantizar el mayor bienestar y 
rendimiento laboral de los empleados. A largo plazo, apoyar a SAW desde perspectivas individuales y organizacionales puede ayudar a mantener vidas laborales más largas y saludables.

\section{Limitaciones y Vías Para Futuras Investigaciones}

La investigación futura debe proporcionar más evidencia de la validez de la escala SAW. Por ejemplo, encontramos una relación positiva significativa de la edad con la tenencia organizacional, pero no encontramos evidencia de diferencias relacionadas con la edad con respecto a las autopercepciones de SAW de los empleados. La falta de evidencia de diferencias relacionadas con la edad puede deberse a que las características de edad de nuestros grupos participantes no se especificaron lo suficientemente bien o porque generalmente no existe un criterio claro y definitivo para dividir a los trabajadores en "mayores" y "más jóvenes" (cf. Zaniboni, Kmicinska, Truxillo, Kahn, Paladino y Fraccaroli, 2019). Además, nuestras muestras piloto y de calibración no incluyeron a ningún participante de más de 60 años de edad. Puede ser el caso de que las personas de 60 años o más, que permanecen en la fuerza laboral, experimenten saw de manera diferente en comparación con los trabajadores a edades más tempranas. La investigación futura debe abordar este problema. Además, es posible que varios moderadores desempeñen un papel en el envejecimiento de los trabajadores (cf. Felicitas, Goecke y Kunze, 2016; Zacher et al., 2018). Es importante destacar que la falta de evidencia de diferencias relacionadas con la edad en las autopercepciones de SAW de los empleados puede indicar que la medida, que sugerimos, captura SAW en todas las etapas de la carrera (cf. Zacher et al., 2018).

Además, en esta etapa del desarrollo de la escala nos centramos en varias características del trabajo y una pequeña gama de variables demográficas. No recopilamos información sobre esas características de trabajo, que pueden estar asociadas negativamente con SAW en algunas etapas de la carrera (por ejemplo, altas demandas físicas en la carrera tardía), las ocupaciones, organizaciones y sectores industriales de los participantes. Dicha información ayudaría a contextualizar y comprender mejor el constructo y la medida de SAW. Además, nos basamos en datos de trabajadores de MTurk, lo que puede limitar la generalización de los resultados del estudio (cf. Walters, Christakis y Wright, 2018). En el futuro, los investigadores de SAW deben tratar de recopilar datos de los trabajadores en todas las etapas de la carrera y en diversos entornos ocupacionales / industriales / culturales con muestras que no sean MTurk. Esto permitirá comparaciones entre grupos de trabajadores en diferentes etapas de carrera/ocupaciones/sectores/culturas. Para comprender mejor la dimensión de edad de SAW, los investigadores deben considerar otros constructos de edad (por ejemplo, edad subjetiva) además de la edad cronológica de los trabajadores (cf. Felicitas et al., 2016) y seguir un enfoque longitudinal (cf. Kooij et al., 2019). Además, en este estudio capturamos las asociaciones entre SAW y solo tres resultados relacionados con el trabajo (es decir, WA y bienestar psicológico, entendido como prosperar en el trabajo y sobrevivir en el trabajo), todos los cuales se autoevaluan. Los datos sobre otros resultados laborales (por ejemplo, el rendimiento de los trabajadores, la salud, otras formas de bienestar, las actitudes laborales, la motivación laboral, las expectativas, etc.; cf. Zacher, 2015a) y de múltiples fuentes serán más valiosos en futuras investigaciones con la escala SAW.

\section{TRANSLATED VERSION: FRENCH}

Below is a rough translation of the insights presented above. This was done to give a general understanding of the ideas presented in the paper. Please excuse any grammatical mistakes and do not hold the original authors responsible for these mistakes.

\section{VERSION TRADUITE: FRANÇAIS}

Voici une traduction approximative des idées présentées ci-dessus. Cela a été fait pour donner une compréhension générale des idées présentées dans le document. Veuillez excuser toutes les erreurs grammaticales et ne pas tenir les auteurs originaux responsables de ces erreurs. 


\section{INTRODUCTION}

Le concept émergent de vieillissement réussi au travail (SAW) est enraciné dans les interprétations contemporaines des théories du développement organisationnel et de la durée de vie. Il met l'accent sur le rôle important des milieux de travail pour la réussite du vieillissement (Olson et Schultz, 2019), ce qui implique que des expériences de travail longues et positives peuvent être maintenues grâce à une adaptation réussie aux changements intra-individuels (p. ex., liés à l'âge) et externes (p. ex., environnementaux) (Kooij, 2015; Olson et Schultz, 2019; Zacher, 2015a). Kooij (2015) a décrit SAW comme le maintien durable de la capacité de travail, de la santé et de la motivation d'un individu tout au long du cycle de vie au travail. Dans cette définition, le rôle actif de l'employé dans la création de ressources de travail et l'assurance d'un adéquation continu entre la personne et l'environnement est d'une importance capitale. Zacher $(2015 \mathrm{a}, \mathrm{b})$ a souligné que SAW ne se limite pas au maintien des niveaux actuels de fonctionnement et peut faire référence au développement ou à la croissance d'un travailleur. Ainsi, saw peut être considéré comme une trajectoire intra-individuelle liée à l'âge vers un résultat de travail (p. ex., bien-être ou rendement au travail) qui s'écarte positivement de la trajectoire moyenne liée à l'âge. Même un employé qui a connu une baisse des résultats au travail au fil du temps peut vieillir avec succès au travail lorsque sa trajectoire est plus positive que la trajectoire moyenne liée à l'âge (Zacher, 2015a, b).

De manière systématique, Zacher, Kooij et Beier (2018) ont décrit les facteurs contribuant au vieillissement actif au travail (AAW), qui est une construction qui chevauche quelque peu SAW (cf. Zacher, 2015a). L'AAW est décrite comme la capacité des travailleurs à « maintenir ou améliorer (1) leur bien-être physique, mental et social; (2) continuer à afficher des niveaux élevés d'engagement et de rendement au travail; et (3) faire l'expérience d'un traitement équitable et de la sécurité d'emploi » dans le processus de vieillissement (Zacher et coll., 2018, p. 37). Parce que le développement est un processus qui dure toute la vie, aAW fait référence aux travailleurs de tous âges et de toutes les étapes de leur carrière. Des facteurs à plusieurs niveaux (individuels, d'équipe, organisationnels et sociétaux au sens large) contribuent à l'AAW/SAW (voir aussi Kooij, Zacher, Wang et Heckhausen, 2019). L'idée de comprendre les facteurs AAW / SAW en relation avec les facteurs internes (par exemple, la personne) et externes (par exemple, environnementaux) est conforme à la critique continue selon laquelle, étant principalement axées sur le processus de développement intra-individuel, les théories de la durée de vie dans leur ensemble sont susceptibles de négliger les effets du contexte sur le développement de l'individu (Olson et Schultz, 2019).

Compte tenu de l'intérêt croissant pour aAW/SAW du point de vue de la durée de vie contemporaine (Zacher et al., 2018), il est surprenant qu'aucune mesure de cette construction n'ait encore été développée. Dans deux études consécutives en 2006 et 2007, Robson et ses collègues ont conceptualisé des critères provisoires pour un vieillissement réussi en milieu de travail et ont élaboré un inventaire pour les mesurer (Robson et Hansson, 2007; Robson, Hansson, Abalos et Booth, 2006). Zacher et Rudolph (2017) ont critiqué ce modèle pour avoir négligé les facteurs contextuels et les résultats du travail en se concentrant entièrement sur les facteurs personnels (stratégies comportementales). En outre, Zacher et Rudolph (2017) ont conclu que parce que les stratégies comportementales suggérées par Robson et al. (Robson \& Hansson, 2007; Robson et coll., 2006) n'interagissent pas avec l'âge, ces stratégies ne peuvent pas être considérées comme des stratégies de vieillissement réussies. Plus récemment, Kooij et ses collègues ont reconnu la difficulté d'opérationnaliser et, par conséquent, de mesurer la construction SAW (Kooij et al., 2019). Comme pour Zacher (2015a), nous comprenons SAW comme un processus de croissance intra-individuelle multidimensionnelle, qui est lié à l'âge, associé à divers critères subjectifs et objectifs, et peut être facilité (ou contraint) par des facteurs à plusieurs niveaux. Sur la base de la définition de Zacher (2015a) de SAW et du cadre des facteurs AAW par Zacher et al. (2018), le but de notre étude est d'étendre les connaissances de SAW en: (a) opérationnalisant des facettes importantes de la construction SAW et (b) en créant une mesure unique de SAW. Nous nous concentrons sur les expériences individuelles et les résultats de SAW, ainsi que sur les antécédents personnels et environnementaux de SAW dans le contexte organisationnel. Nous considérons également les interactions potentielles de ces facteurs avec l'âge des travailleurs (cf. Zacher et al., 2015a, 2018). Nous testons les propriétés psychométriques de la nouvelle mesure dans trois échantillons MTurk consécutifs et suggérons des directions pour les recherches futures. 


\section{Opérationnaliser le Vieillissement Réussi au Travail \\ Expériences des Employés de SAW}

Les impacts des changements liés à l'âge sur les comportements et les résultats au travail (en particulier en fin de carrière) sont largement documentés. La nature et l'étendue des changements liés à l'âge peuvent varier considérablement d'une personne à l'autre en raison de différences dans les facteurs personnels et environnementaux. Ces changements (p. ex., les capacités cognitives, la personnalité et la motivation des travailleurs) sont susceptibles de refléter diverses pertes et gains de développement tout au long du cycle de vie au travail (Kanfer et Ackerman, 2004; Warr, 2001). Récemment, le nombre d'études axées sur les aspects positifs (c.-à-d. les gains de développement) par opposition aux aspects liés aux pertes (c.-à-d. les déclins du développement) du processus de vieillissement a augmenté. Par exemple, dans une étude qualitative menée auprès de 37 travailleurs âgés (âgés de 55 ans et plus) de deux pays (Bulgarie et RoyaumeUni) et de deux secteurs industriels (soins de santé et technologies de l'information et de la communication), Taneva, Arnold et Nicolson (2016) ont constaté que les travailleurs en fin de carrière sont bien conscients de la façon dont les changements liés à l'âge peuvent affecter leur vie professionnelle et leurs résultats. Il est important de savoir si les travailleurs associent certains de ces changements (p. ex., amélioration des connaissances et de l'expérience, amélioration de l'expérience sociale et émotionnelle) à leur développement personnel et professionnel et, en fin de compte, à l'amélioration des résultats au travail. Ainsi, les perceptions des travailleurs à l'égard des changements positifs liés à l'âge (gains de développement; cf. Kanfer et Ackerman, 2004) sont susceptibles d'indiquer les expériences des travailleurs de SAW.

Dans l'étude de Taneva et al. (2016), parallèlement à leurs perceptions d'une connaissance et d'une expérience accrues, les travailleurs (âgés) ont communiqué leurs perceptions d'autres domaines de croissance personnelle et professionnelle, parmi lesquels une conscience de soi et une confiance accrues, une amélioration des compétences sociales, une régulation des émotions, une capacité à résoudre des problèmes complexes et une adaptation générale aux changements contextuels. Ces résultats, extraits des récits des travailleurs, correspondent aux résultats de recherches antérieures sur la tradition de la durée de vie (p. ex., Carstensen et coll., 2011; Kanfer et Ackerman, 2004). Nous suggérons que les conceptualisations par les travailleurs de changements intra-individuels positifs peuvent façonner un domaine important de la construction SAW.

Stratégies Personnelles et Organisationnelles Pour SAW

Du point de vue de la théorie de la durée de vie, le vieillissement est un processus proactif d'adaptation, motivé par l'autorégulation sous la forme de stratégies de gestion de la vie que les individus appliquent afin de faire face aux changements liés à l'âge (par exemple, perte, gain et / ou réorganisation des ressources) en eux-mêmes ainsi qu'aux changements dans leur environnement (Kanfer et Ackerman, 2004). Le modèle d'optimisation sélective avec compensation (SOC) (Baltes \& Baltes, 1990) propose que les individus sont susceptibles d'utiliser des stratégies qui se répartissent en trois catégories de processus d'autorégulation (sélection, optimisation et compensation) dans leurs tentatives de s'adapter à la fois aux changements liés à l'âge et aux changements dans leur environnement. Ces comportements proactifs deviennent plus importants avec l'âge et peuvent être particulièrement utiles sur le lieu de travail. En commençant par les travaux pionniers d'Abraham et Hansson (1995), une pléthore d'études a démontré les effets positifs des stratégies SOC sur le bien-être et la performance des travailleurs (âgés) (par exemple, Moghimi, Scheibe et Freund, 2019; Moghimi, Zacher, Shreible, \& Van Yperen, 2017). Ainsi, les stratégies SOC peuvent être un mécanisme efficace pour atteindre SAW.

En combinant les avantages de la conception des emplois et des théories de la durée de vie, Truxillo, Cadiz, Rineer, Zaniboni et Fraccaroli (2012) ont proposé des effets potentiels liés à l'âge de trois sousgroupes de caractéristiques de l'emploi (tâche, connaissances et social) sur l'engagement au travail, la satisfaction au travail et le rendement au travail. Par exemple, ils ont suggéré qu'en raison des changements intra-individuels liés à l'âge, certaines caractéristiques de l'emploi telles que l'autonomie professionnelle, l'importance des tâches, la variété des compétences et le soutien social peuvent être préférées par les travailleurs plus âgés (par rapport aux plus jeunes) et, par conséquent, faciliter des résultats professionnels positifs, en particulier en fin de carrière. Entre autres, Zacher et al. (2018) ont souligné l'importance de 
l'autonomie au travail, de l'intégration sociale et du sens du sens pour les travailleurs vieillissants. Par conséquent, permettre des possibilités d'autonomie professionnelle plus élevée, d'importance des tâches, de variété de compétences et de soutien social peut favoriser SAW tout au long de la vie (voir aussi Cadix, Rineer et Truxillo, 2019).

Les approches formelles (p. ex., gestion stratégique des ressources humaines) et informelles (p. ex., ideals entre les employés et leurs superviseurs) de gestion des ressources humaines (GRH) se sont avérées importantes pour soutenir le bien-être et le rendement des travailleurs (Bal, 2015; Kooij, Jansen, Dikkers et De Lange, 2014). Certaines pratiques formelles de GRH (également appelées « amies des aînés », cf. Zacher et coll., 2018) sont considérées comme ayant des effets souhaitables, en particulier pour les travailleurs âgés. Kooij et coll. (2014) ont conceptualisé quatre ensembles de pratiques de GRH pour les travailleurs vieillissants, dont des pratiques d'entretien et de perfectionnement. Taneva et Arnold (2018) ont rapporté qu'une combinaison spécifique entre certaines pratiques de GRH d'entretien et de développement prédisait des expériences de bien-être au travail élevé (épanouissement au travail) et de rendement professionnel élevé chez un large échantillon de travailleurs âgés au Royaume-Uni. Ces pratiques, les plus appréciées par les travailleurs âgés, font référence à l'accès à la formation, à la reconnaissance et au respect, à des affectations significatives, à la rétroaction du superviseur, à la flexibilité du travail et aux possibilités de travailler plus longtemps. En outre, il existe de nombreuses preuves de la valeur des modalités de travail informelles et du rôle des superviseurs en fin de carrière. Par exemple, Bal (2015) a conclu que " l'individualisation des arrangements de carrière sera de plus en plus importante dans la durabilité des carrières contemporaines » (Bal, 2015, p. 1). Sur la base de ces preuves, nous suggérons que l'accès des travailleurs à une combinaison de certaines pratiques de GRH avec des accords de travail informels (ideals) peut être déterminant pour atteindre saw dans les organisations.

\section{Résultats d'un Vieillissement Réussi au Travail}

Théoriquement, SAW a été positivement associé à divers résultats de travail au niveau individuel, parmi lesquels la motivation au travail, le rendement au travail, la santé et le bien-être (Zacher, 2015a). Jusqu'à présent, seules quelques études ont fourni un soutien empirique à certaines de ces associations. Par exemple, Taneva et Arnold (2018) ont signalé trois voies d'accès à saw dans les organisations qui démontrent le rôle des stratégies personnelles (SOC) et organisationnelles (HRM) pour atteindre un rendement élevé au travail. De plus, ils ont constaté que le bien-être psychologique des employés médiait les relations entre les stratégies personnelles et organisationnelles et le rendement au travail autoéconït (proactivité des tâches, rendement extra et en cours de rôle). Par conséquent, le bien-être des travailleurs est un résultat important et, peut-être plus direct (par rapport au rendement au travail), de SAW.

S'épanouir au travail est décrit comme « le sentiment commun de vitalité et d'apprentissage, qui communique un sentiment de progrès ou de mouvement vers l'avant dans son développement personnel » (Spreitzer, Sutcliffe, Dutton, Sonenshein et Grant, 2005, p. 538). Les expériences d'épanouissement au travail peuvent être motivées par des facteurs personnels et organisationnels (Spreizer, Porath et Gibson, 2012). S'épanouir au travail est positivement lié à des résultats tels que le rendement élevé au travail et dans la carrière, les comportements positifs au travail, le bien-être lié au travail et le bien-être général (Porath, Spreitzer, Gibson et Garnett, 2012). Deux études de Taneva et de ses collègues (Taneva et coll., 2016; Taneva \& Arnold, 2018) ont démontré que des niveaux élevés de prospérité au travail sont également fréquents chez les travailleurs âgés. Par conséquent, des expériences d'épanouissement au travail peuvent se produire à toutes les étapes de la carrière. De plus, s'épanouir au travail peut être considéré à la fois comme une forme de bien-être psychologique (cf. Spreitzer et al., 2005; Warr, 2007) et un critère pour SAW (Taneva \& Arnold, 2018).

Par opposition à la prospérité, la construction émergente de la survie au travail est décrite comme la tendance de l'individu à préserver ses ressources mentales et physiques en limitant ses activités et ses perspectives de travail pour faire face à des conditions de travail perçues comme très exigeantes (Taneva et al., 2016). Une telle expérience de préservation peut indiquer des niveaux réduits ou un manque de SAW. Cependant, Taneva et Arnold (2018) ont découvert que, du moins dans certaines circonstances, les expériences de survie au travail peuvent en effet être un signe d'adéquation durable entre la personne et 
l'environnement et, en fin de compte, de SAW. Cela est également conforme à la définition de Kooij de saw en tant que maintenance durable (Kooij, 2015) et à la définition de l'AAW par Zacker et ses collègues (Zacher et al., 2018). Par conséquent, survivre au travail peut être un autre indicateur de SAW.

De plus, comme il est indiqué dans les sections précédentes, l'expérience des individus à l'égard de SAW peut être améliorée à l'aide de stratégies personnelles (p. ex., SOC) ou organisationnelles (p. ex., GRH, i-deals et conception d'emplois) qui facilitent la réglementation de leurs ressources. Le concept de capacité de travail (WA) décrit l'équilibre entre le travail et les ressources personnelles (Tuomi, Ilmarinen, Jahkola, Katajarinne et Tulkki, 1998). Des recherches récentes sur la durée de vie ont démontré que certaines caractéristiques du travail (p. ex., le contrôle de l'emploi, la rétroaction et le soutien social) et de la personne (p. ex., comportements proactifs) peuvent soutenir le maintien et même l'augmentation de l'AO, en particulier en fin de carrière (p. ex., Weigl, Müller, Hornung, Zacher et Angerer, 2013). Dans ce contexte, il a été suggéré que l'AO pourrait être un critère utile pour saw en fin de carrière (et au-delà) (Kooij, 2015; Weigl et coll., 2013).

\section{CONCLUSION}

Nous avons développé et établi les propriétés psychométriques de la première mesure unique pour SAW dans le sens contemporain de cette construction (cf. Zacher, 2015a). Nous considérons SAW comme un processus de développement qui peut se produire à toutes les étapes de la carrière et peut être capturé par des résultats au niveau individuel tels que l'amélioration de l'AO et du bien-être. Nous avons opérationnalisé la construction SAW à travers deux groupes de perceptions des employés: (a) les expériences psychologiques des individus de la croissance liée à l'âge en milieu de travail (SAW), et (b) les stratégies pour atteindre SAW. Les expériences de croissance peuvent faire référence à divers domaines de changements positifs perçus liés à l'âge, parmi lesquels l'amélioration des connaissances et de l'expérience, les compétences sociales, la régulation des émotions et l'adaptabilité dans les situations liées au travail (cf. Taneva et al., 2016). Les stratégies (ou facilitateurs) de SAW peuvent inclure à la fois des comportements des employés (p. ex., autoréglementation proactive) et organisationnels (p. ex., conception des tâches, GRH formelle et arrangements de travail informels) (cf. Bal, 2015; Baltes \& Baltes, 1990; Kooij et coll., 2014; Taneva et coll., 2016; Truxillo et coll., 2012).

Une analyse empirique subséquente avec les données de trois échantillons consécutifs de MTurk avec un total de 417 travailleurs en milieu organisationnel a révélé que les expériences des employés de SAW chevauchaient largement les perceptions des employés à l'égard des facilitateurs individuels de SAW (c.à-d. stratégies personnelles). Une explication possible pourrait être que saw pourrait également être décrit comme un processus d'autorégulation active, motivé par la motivation de l'individu à poursuivre l'adéquation personne-environnement (Kooij et al., 2019). Ainsi, les perceptions des employés à la fois des changements positifs liés à l'âge et des comportements d'autorégulation proactifs (stratégies personnelles) peuvent refléter la construction SAW dans le sens d'une croissance perçue liée à l'âge (cf. Taneva et al., 2016).

En outre, nous avons examiné la validité de la nouvelle échelle liée à la construction et aux critères en explorant les associations du score total de l'échelle SAW avec les scores de six mesures établies des stratégies personnelles (SOC) et organisationnelles (conception des emplois, GRH formelle), ainsi que des critères subjectifs pour SAW (WA, prospérer et survivre au travail). Les analyses empiriques ont confirmé toutes les relations anticipées, à l'exception de l'association entre le score SAW et le score Surviving at Work, qui semblait manquer de signification statistique. C'est peut-être parce que nous avons suggéré de survivre au travail comme critère provisoire pour SAW. Bien que l'expérience de survie des employés au travail puisse indiquer le maintien des ressources et donc des résultats de travail durables (p. ex., le bienêtre) (cf. Kooij, 2015; Taneva \& Arnold, 2018), la construction survivre au travail n'implique pas de croissance perçue comme c'est le cas avec SAW dans la définition de Zacher (cf. Zacher, 2015a). 


\section{Contributions à la Théorie et À La Pratique}

Cette étude a contribué au développement de la théorie de plusieurs façons. Tout d'abord, il a systénalisé et conceptualisé les domaines clés de la construction émergente SAW en reliant les variables personnelles et environnementales. Ainsi, il a répondu aux appels récents à l'intégration de facteurs à plusieurs niveaux lors de l'examen de cette construction (cf. Zacher, 2015a; Zacher et coll., 2018). Deuxièmement, il s'est concentré sur les synergies entre ces domaines et a acquis de nouvelles connaissances sur la façon dont ils fonctionnent ensemble, ainsi que séparément. Troisièmement, il a traduit en termes pratiques chaque domaine et a exploré sa construction et sa validité liée aux critères en ce qui concerne d'autres constructions bien connues et des variables démographiques. Ainsi, de nouvelles informations sur le contenu de chaque domaine ont été trouvées et un nouvel instrument de mesure a été développé. Du point de vue du praticien, cet instrument aidera les professionnels de la formation et du développement à saisir les expériences des travailleurs et leur potentiel à vieillir avec succès dans les organisations. De plus, cette échelle peut aider les travailleurs à surveiller et à diriger leurs propres expériences de SAW. Ceci, à son tour, peut éclairer les systèmes organisationnels et les initiatives visant à soutenir SAW et donc assurer le bien-être et le rendement au travail plus élevés des employés. À plus long terme, le soutien de SAW d'un point de vue individuel et organisationnel peut aider à maintenir une vie professionnelle plus longue et plus saine.

\section{Limites et Avenues Pour La Recherche Future}

Les recherches futures doivent fournir des preuves supplémentaires de la validité de l'échelle SAW. Par exemple, nous avons constaté une relation positive significative entre l'âge et la permanence organisationnelle, mais nous n'avons pas trouvé de preuves de différences liées à l'âge concernant la perception que les employés ont d'eux-mêmes de saw. L'absence de preuves de différences liées à l'âge peut être due au fait que les caractéristiques d'âge de nos groupes de participants n'ont pas été suffisamment bien spécifiées ou parce qu'il n'y a généralement pas de critère clair et définitif pour diviser les travailleurs en «plus âgés » et «plus jeunes » (cf. Zaniboni, Kmicinska, Truxillo, Kahn, Paladino et Fraccaroli, 2019). De plus, nos échantillons pilotes et d'étalonnage n'incluaient aucun participant de plus de 60 ans. Il se peut que les personnes âgées de 60 ans et plus, qui restent sur le marché du travail, vivent saw différemment par rapport aux travailleurs plus jeunes. Les recherches futures devraient aborder cette question. En outre, il est possible que divers modérateurs jouent un rôle dans le vieillissement des travailleurs (cf. Felicitas, Goecke et Kunze, 2016; Zacher et coll., 2018). Il est important de constater que l'absence de preuves de différences liées à l'âge dans la perception de soi des employés à l'égard de saw peut indiquer que la mesure, que nous suggérons, englobe saw à toutes les étapes de leur carrière (cf. Zacher et al., 2018).

De plus, à ce stade du développement de l'échelle, nous nous sommes concentrés sur plusieurs caractéristiques de l'emploi et un petit éventail de variables démographiques. Nous n'avons pas recueilli d'information sur ces caractéristiques de travail, qui peuvent être associées négativement à saw à certaines étapes de la carrière (p. ex., exigences physiques élevées en fin de carrière), les professions, les organisations et les secteurs industriels des participants. De telles informations aideraient à contextualiser et à mieux comprendre la construction et la mesure saw. De plus, nous nous sommes appuyés sur les données des travailleurs de MTurk, ce qui peut limiter la généralisabilité des résultats de l'étude (cf. Walters, Christakis et Wright, 2018). À l'avenir, les chercheurs de SAW devraient chercher à recueillir des données auprès des travailleurs à tous les stades de leur carrière et dans divers contextes professionnels/ industriels/culturels avec des échantillons non-MTurk. Cela permettra des comparaisons entre des groupes de travailleurs à différents stades de carrière/ professions / secteurs / cultures. Pour mieux comprendre la dimension âge de saw, les chercheurs devraient considérer d'autres concepts d'âge (p. ex., l'âge subjectif) en plus de l'âge chronologique des travailleurs (cf. Felicitas et coll., 2016) et suivre une approche longitudinale (cf. Kooij et coll., 2019). De plus, dans cette étude, nous avons saisi les associations entre saw et seulement trois résultats liés au travail (c.-à-d. l'AO et le bien-être psychologique, compris comme étant à la fois s'épanouir au travail et survivre au travail), qui sont tous auto-évalués. Les données concernant d'autres résultats au travail (p. ex., le rendement des travailleurs, la santé, d'autres formes de 
bien-être, les attitudes au travail, la motivation au travail, les attentes, etc.; cf. Zacher, 2015a) et provenant de sources multiples seront les plus précieuses dans les recherches futures avec l'échelle SAW.

\section{TRANSLATED VERSION: GERMAN}

Below is a rough translation of the insights presented above. This was done to give a general understanding of the ideas presented in the paper. Please excuse any grammatical mistakes and do not hold the original authors responsible for these mistakes.

\section{ÜBERSETZTE VERSION: DEUTSCH}

Hier ist eine ungefähre Übersetzung der oben vorgestellten Ideen. Dies wurde getan, um ein allgemeines Verständnis der in dem Dokument vorgestellten Ideen zu vermitteln. Bitte entschuldigen Sie alle grammatikalischen Fehler und machen Sie die ursprünglichen Autoren nicht für diese Fehler verantwortlich.

\section{EINLEITUNG}

Das aufkommende Konzept des erfolgreichen Alterns bei der Arbeit (SAW) wurzelt in den zeitgenössischen Interpretationen der Organisations- und Lebensspannenentwicklungstheorien. Es betont die wichtige Rolle von Arbeitsplätzen für erfolgreiches Altern (Olson \& Schultz, 2019) und impliziert, dass lange und positive arbeitsbezogene Erfahrungen durch eine erfolgreiche Anpassung an intra-individuelle (z. B. altersbedingte) und externe (z. B. umweltbedingte) Veränderungen aufrechterhalten werden können (Kooij, 2015; Olson \& Schultz, 2019; Zacher, 2015a). Kooij (2015) beschrieb SAW als die nachhaltige Erhaltung der Arbeitsfähigkeit, Gesundheit und Motivation eines Individuums über den gesamten Arbeitslebenszyklus. In dieser Definition ist die aktive Rolle des Mitarbeiters für die Schaffung von Arbeitsressourcen und die Sicherstellung einer kontinuierlichen Person-Environment-Fit von zentraler Bedeutung. Zacher (2015a, b) wies darauf hin, dass SAW nicht auf die Aufrechterhaltung des aktuellen Funktionsniveaus beschränkt ist und sich auf die Entwicklung oder das Wachstum eines Arbeitnehmers beziehen kann. Somit kann SAW als intra-individuelle altersbedingte Trajektorie zu einem Arbeitsergebnis (z. B. Wohlbefinden oder Arbeitsleistung) betrachtet werden, das positiv von der durchschnittlichen altersbedingten Trajektorie abweicht. Selbst ein Mitarbeiter, der im Laufe der Zeit einen Rückgang eines Arbeitsergebnisses erlebt hat, kann bei der Arbeit erfolgreich altern, wenn seine / ihre Entwicklung positiver ist als die durchschnittliche altersbedingte Entwicklung (Zacher, 2015a, b).

Zacher, Kooij und Beier (2018) skizzierten systematisch die Faktoren, die zum aktiven Altern bei der Arbeit (AAW) beitragen, ein Konstrukt, das sich etwas mit SAW überschneidet (vgl. Zacher, 2015a). AAW wird als die Fähigkeit der Arbeitnehmer beschrieben, "ihr körperliches, geistiges und soziales Wohlbefinden zu erhalten oder zu verbessern; (2) weiterhin ein hohes Maß an Arbeitsengagement und leistung aufweisen; und (3) faire Behandlung und Beschäftigungssicherheit erleben" im Alterungsprozess (Zacher et al., 2018, S. 37). Da Entwicklung ein lebenslanger Prozess ist, bezieht sich AAW auf Arbeitnehmer jeden Alters und jeder Karrierestufe. Mehrstufige Faktoren (Einzel-, Team-, Organisationsund breitere gesellschaftliche Faktoren) tragen zu AAW / SAW bei (siehe auch Kooij, Zacher, Wang \& Heckhausen, 2019). Die Idee, AAW / SAW sowohl in Bezug auf interne (z. B. Person) als auch externe (z. B. Umwelt) Faktoren $\mathrm{zu}$ verstehen, steht im Einklang mit der anhaltenden Kritik, dass Lebensspannentheorien, die sich hauptsächlich auf den Prozess der intra-individuellen Entwicklung konzentrieren, die Auswirkungen des Kontexts auf die Entwicklung des Individuums wahrscheinlich vernachlässigen (Olson \& Schultz, 2019).

Angesichts des zunehmenden Interesses an AAW/SAW aus einer zeitgenössischen Lebensperspektive (Zacher et al., 2018) ist es verwunderlich, dass noch kein Maß für dieses Konstrukt entwickelt wurde. In zwei aufeinanderfolgenden Studien in den Jahren 2006 und 2007 konzipierten Robson und seine Kollegen vorläufige Kriterien für erfolgreiches Altern am Arbeitsplatz und entwickelten ein Inventar zu deren 
Messung (Robson \& Hansson, 2007; Robson, Hansson, Abalos \& Booth, 2006). Zacher \& Rudolph (2017) kritisierten dieses Modell, weil es die kontextuellen Faktoren und Arbeitsergebnisse vernachlässigt, indem es sich ganz auf Personenfaktoren (Verhaltensstrategien) konzentriert. Darüber hinaus kamen Zacher \& Rudolph (2017) zu dem Schluss, dass die von Robson et al. vorgeschlagenen Verhaltensstrategien (Robson \& Hansson, 2007; Robson et al., 2006) interagieren nicht mit dem Alter, diese Strategien können nicht als erfolgreiche Alterungsstrategien angesehen werden. Zuletzt erkannten Kooij und Kollegen die Schwierigkeit an, das Konstrukt SAW zu operationalisieren und damit zu messen (Kooij et al., 2019). Wie bei Zacher (2015a) verstehen wir SAW als einen Prozess des multidimensionalen intra-individuellen Wachstums, der altersbedingt ist, mit verschiedenen subjektiven und objektiven Kriterien verbunden ist und durch mehrstufige Faktoren erleichtert (oder eingeschränkt) werden kann. Basierend auf Zachers (2015a) Definition von SAW und dem Framework der AAW-Faktoren von Zacher et al. (2018) besteht der Zweck unserer Studie darin, das Wissen über SAW zu erweitern, indem wir: (a) wichtige Facetten des Konstrukts SAW operationalisieren und (b) ein einzelnes Maß von SAW erstellen. Wir konzentrieren uns auf die individuellen Erfahrungen und Ergebnisse von SAW sowie auf personen- und umweltbedingte Vorläufer von SAW im organisatorischen Kontext. Wir berücksichtigen auch die möglichen Wechselwirkungen dieser Faktoren mit dem Alter der Arbeitnehmer (vgl. Zacher et al., 2015a, 2018). Wir testen die psychometrischen Eigenschaften der neuen Maßnahme in drei aufeinanderfolgenden MTurkProben und schlagen Richtungen für die zukünftige Forschung vor.

\section{Operationalisierung des Erfolgreichen Alterns bei der Arbeit Erfahrungen der Mitarbeiter mit SAW}

Die Auswirkungen altersbedingter Veränderungen auf das Arbeitsverhalten und die Arbeitsergebnisse (insbesondere in der späten Karriere) sind ausführlich dokumentiert. Art und Ausmaß altersbedingter Veränderungen können aufgrund von Unterschieden in Person und Umweltfaktoren zwischen Individuen erheblich variieren. Diese Veränderungen (z. B. in den kognitiven Fähigkeiten, der Persönlichkeit und der Motivation der Arbeitnehmer) spiegeln wahrscheinlich verschiedene Entwicklungsverluste und -gewinne über den gesamten Arbeitslebenszyklus wider (Kanfer \& Ackerman, 2004; Warr, 2001). In jüngster Zeit hat die Anzahl der Studien, die sich auf die positiven (d. H. Entwicklungsgewinne) im Gegensatz zu verlustbedingten (d. H. Entwicklungsrückgängen) Aspekten des Alterungsprozesses konzentrierten, zugenommen. In einer qualitativen Studie mit 37 älteren Arbeitnehmern (im Alter von 55 Jahren und älter) aus zwei Ländern (Bulgarien und Großbritannien) und zwei Industriesektoren (Gesundheitswesen und Informations- und Kommunikationstechnologien) fanden Taneva, Arnold und Nicolson (2016) heraus, dass Arbeitnehmer mit später Laufbahn sich bewusst sind, wie sich altersbedingte Veränderungen auf ihr Arbeitsleben und ihre Ergebnisse auswirken können. Wichtig ist, dass Arbeitnehmer einige dieser Veränderungen (z. B. mehr Wissen und Erfahrung, verbesserte soziale und emotionale Erfahrung) mit ihrer persönlichen und beruflichen Entwicklung und letztendlich mit verbesserten Arbeitsergebnissen in Verbindung bringen. Daher ist es wahrscheinlich, dass die Wahrnehmung positiver altersbedingter Veränderungen durch die Arbeitnehmer (Entwicklungsgewinne; vgl. Kanfer \& Ackerman, 2004) auf die Erfahrungen der Arbeitnehmer mit SAW hinweist.

In der Studie von Taneva et al. (2016) kommunizierten (ältere) Arbeitnehmer zusammen mit ihrer Wahrnehmung von erhöhtem Wissen und Erfahrung Wahrnehmungen anderer Bereiche des persönlichen und beruflichen Wachstums, darunter erhöhtes Selbstbewusstsein und Selbstvertrauen, verbesserte soziale Fähigkeiten, Emotionsregulation, Fähigkeit, komplexe Probleme zu lösen und allgemeine Anpassung an kontextuelle Veränderungen. Diese Ergebnisse, die aus den Erzählungen der Arbeiter extrahiert wurden, stimmen mit den Ergebnissen früherer Forschungen in der Lebensspannentradition überein (z. B. Carstensen et al., 2011; Kanfer \& Ackerman, 2004). Wir schlagen vor, dass die Konzeptualisierungen positiver intrainterner Veränderungen durch die Arbeiter eine wichtige Domäne des Konstrukts SAW prägen können. 


\section{Persönliche und Organisatorische Strategien für SAW}

Aus lebensspannentheoretischer Sicht ist das Altern ein proaktiver Anpassungsprozess, der durch Selbstregulierung in Form von Lebensmanagementstrategien angetrieben wird, die Individuen anwenden, um mit altersbedingten Veränderungen (z. B. Verlust, Gewinn und / oder Reorganisation von Ressourcen) an sich sowie Veränderungen in ihrer Umgebung umzugehen (Kanfer \& Ackerman, 2004). Das Modell der selektiven Optimierung mit Kompensation (SOC) (Baltes \& Baltes, 1990) schlägt vor, dass Individuen wahrscheinlich Strategien verwenden, die in drei Kategorien von Selbstregulierungsprozessen (Selektion, Optimierung und Kompensation) fallen, um sich sowohl an altersbedingte Veränderungen als auch an Veränderungen in ihrer Umgebung anzupassen. Diese proaktiven Verhaltensweisen werden mit zunehmendem Alter wichtiger und können besonders am Arbeitsplatz nützlich sein. Beginnend mit der Pionierarbeit von Abraham und Hansson (1995) hat eine Vielzahl von Studien die positiven Auswirkungen von SOC-Strategien auf das Wohlbefinden und die Leistung (älterer) Arbeitnehmer gezeigt (z. B. Moghimi, Scheibe \& Freund, 2019; Moghimi, Zacher, Shreible\& Van Yperen, 2017). Daher können SOC-Strategien ein effektiver Mechanismus sein, um SAW zu erreichen.

Truxillo, Cadiz, Rineer, Zaniboni und Fraccaroli (2012) kombinierten die Vorteile der Jobdesign- und Lebensdauertheorien und schlugen mögliche altersbedingte Auswirkungen von drei Untergruppen von Arbeitsmerkmalen (Aufgabe, Wissen und Soziales) auf das Arbeitsengagement, die Arbeitszufriedenheit und die Arbeitsleistung vor. Zum Beispiel schlugen sie vor, dass aufgrund intra-individueller altersbedingter Veränderungen einige Arbeitsplatzmerkmale wie Arbeitsplatzautonomie, Aufgabenbedeutung, Kompetenzvielfalt und soziale Unterstützung von älteren (im Vergleich zu jüngeren) Arbeitnehmern bevorzugt werden können und daher positive Arbeitsergebnisse insbesondere in der späten Karriere ermöglichen. Zacher et al. (2018) betonten unter anderem die Bedeutung von Arbeitsautonomie, sozialer Einbettung und Sinn für alternde Arbeitnehmer. Daher kann das Zulassen von Möglichkeiten für eine höhere berufliche Autonomie, Aufgabenbedeutung, Kompetenzvielfalt und soziale Unterstützung SAW über die gesamte Lebensspanne hinweg fördern (siehe auch Cadiz, Rineer \& Truxillo, 2019).

Sowohl formelle (z. B. strategisches Personalmanagement) als auch informelle (z. B. I-Deals zwischen Mitarbeitern und ihren Vorgesetzten) Human Resource Management (HRM) Ansätze erweisen sich als wichtig für die Unterstützung des Wohlbefindens und der Leistung der Arbeitnehmer (Bal, 2015; Kooij, Jansen, Dikkers\& De Lange, 2014). Einige formale HRM-Praktiken (auch als "altersfreundlich" bezeichnet, vgl. Zacher et al., 2018) werden als wünschenswerte Wirkungen insbesondere für ältere Arbeitnehmer angesehen. Kooij et al. (2014) konzipierten vier Bündel von HRM-Praktiken für alternde Arbeitnehmer, darunter Wartungs- und Entwicklungspraktiken. Taneva und Arnold (2018) berichteten, dass eine spezifische Kombination zwischen einigen HRM-Praktiken in der Instandhaltung und Entwicklung bei einer großen Stichprobe älterer Arbeitnehmer im Vereinigten Königreich Erfahrungen mit hohem Wohlbefinden bei der Arbeit (Gedeihen bei der Arbeit) und hoher Arbeitsleistung vorhersagte. Diese Praktiken, die von den älteren Arbeitnehmern am meisten geschätzt werden, beziehen sich auf den Zugang zu Ausbildung, Anerkennung und Respekt, sinnvolle Aufgaben, Feedback des Vorgesetzten, flexibles Arbeiten und Möglichkeiten, länger zu arbeiten. Darüber hinaus gibt es erhebliche Beweise für den Wert informeller Arbeitsvereinbarungen und die Rolle von Vorgesetzten in der späten Karriere. Zum Beispiel kam Bal (2015) zu dem Schluss, dass "die Individualisierung von Karrieregestaltungen für die Nachhaltigkeit zeitgenössischer Karrieren immer wichtiger wird" (Bal, 2015, S. 1). Basierend auf diesen Erkenntnissen schlagen wir vor, dass der Zugang von Arbeitnehmern zu einer Kombination bestimmter HRM-Praktiken mit informellen Arbeitsvereinbarungen (i-Deals) für das Erreichen von SAW in Organisationen von entscheidender Bedeutung sein kann.

\section{Ergebnisse des Erfolgreichen Alterns bei der Arbeit}

Theoretisch wurde SAW positiv mit verschiedenen Arbeitsergebnissen auf individueller Ebene in Verbindung gebracht, darunter Arbeitsmotivation, Arbeitsleistung, Gesundheit und Wohlbefinden (Zacher, 2015a). Bisher haben nur wenige Studien empirische Unterstützung für einige dieser Assoziationen geliefert. Zum Beispiel berichteten Taneva und Arnold (2018) über drei Wege zu SAW in Organisationen, die die Rolle persönlicher (SOC) und organisatorischer (HRM) Strategien für die Erreichung einer hohen 
Arbeitsleistung demonstrieren. Darüber hinaus fanden sie heraus, dass das psychische Wohlbefinden der Mitarbeiter die Beziehungen zwischen persönlichen und organisatorischen Strategien und der selbst bewerteten Arbeitsleistung (Aufgabenproaktivität, Außer- und In-Rollen-Leistung) vermittelte. Daher ist das Wohlbefinden der Arbeitnehmer ein wichtiges und vielleicht direkteres (im Vergleich zur Arbeitsleistung) Ergebnis von SAW.

Gedeihen bei der Arbeit wird beschrieben als "das gemeinsame Gefühl von Vitalität und Lernen, das ein Gefühl des Fortschritts oder der Vorwärtsbewegung in der eigenen Selbstentwicklung vermittelt" (Spreitzer, Sutcliffe, Dutton, Sonenshein, \& Grant, 2005, S. 538). Erfahrungen mit dem Gedeihen bei der Arbeit können sowohl von personischen als auch von organisatorischen Faktoren angetrieben werden (Spreizer, Porath , \& Gibson, 2012). Das Gedeihen bei der Arbeit hängt positiv mit Ergebnissen wie hoher Arbeits- und Karriererollenleistung, positivem Arbeitsverhalten, arbeitsbezogenem und allgemeinem Wohlbefinden zusammen (Porath, Spreitzer, Gibson \& Garnett, 2012). Zwei Studien von Taneva und Kollegen (Taneva et al., 2016; Taneva \& Arnold, 2018) zeigten, dass ein hohes Maß an Gedeihen bei der Arbeit auch bei älteren Arbeitnehmern üblich ist. Daher können Erfahrungen mit dem Gedeihen bei der Arbeit in allen Karrierephasen stattfinden. Darüber hinaus kann das Gedeihen am Arbeitsplatz sowohl als eine Form des psychischen Wohlbefindens angesehen werden (vgl. Spreitzer et al., 2005; Warr, 2007) und ein Kriterium für SAW (Taneva \& Arnold, 2018).

Im Gegensatz zum Gedeihen wird das aufkommende Konstrukt des Überlebens bei der Arbeit als die Tendenz des Individuums beschrieben, seine geistigen und körperlichen Ressourcen zu erhalten, indem es seine Arbeitsaktivitäten und Perspektiven einschränkt, um mit Arbeitsbedingungen fertig zu werden, die als sehr anspruchsvoll wahrgenommen werden (Taneva et al., 2016). Eine solche Konservierungserfahrung kann auf reduzierte Werte oder einen Mangel an SAW hinweisen. Taneva und Arnold (2018) entdeckten jedoch, dass zumindest unter bestimmten Umständen Erfahrungen des Überlebens bei der Arbeit tatsächlich ein Zeichen für eine anhaltende Mensch-Umwelt-Fit und letztendlich für SAW sein können. Dies steht auch im Einklang mit Kooijs Definition von SAW als nachhaltige Instandhaltung (Kooij, 2015) und der Definition von AAW von Zacker und Kollegen (Zacher et al., 2018). Daher kann das Überleben bei der Arbeit ein weiterer Indikator für SAW sein.

Darüber hinaus können, wie in den vorherigen Abschnitten beschrieben, die Erfahrungen von Einzelpersonen mit SAW durch persönliche (z. B. SOC) oder organisatorische (z. B. HRM, i-Deals und Job-Design) Strategien verbessert werden, die die Regulierung der eigenen Ressourcen erleichtern. Das Konzept der Arbeitsfähigkeit (WA) beschreibt das Gleichgewicht (Fit) zwischen Arbeit und persönlichen Ressourcen (Tuomi, Ilmarinen, Jahkola, Katajarinne, \& Tulkki, 1998). Jüngste Lebensspannenforschungen haben gezeigt, dass bestimmte Arbeits- (z. B. Arbeitsplatzkontrolle, Feedback und soziale Unterstützung) und Personenmerkmale (z. B. proaktives Verhalten) die Aufrechterhaltung und sogar die Zunahme von WA unterstützen können, insbesondere in der späten Karriere (z. B. Weigl, Müller, Hornung, Zacher \& Angerer, 2013). In diesem Zusammenhang wurde vorgeschlagen, dass WA ein nützliches Kriterium für SAW in der späten Karriere (und darüber hinaus) sein könnte (Kooij, 2015; Weigl et al., 2013).

\section{SCHLUSSFOLGERUNG}

Wir entwickelten und etablierten die psychometrischen Eigenschaften des ersten Einzelmaßes für SAW in der zeitgenössischen Bedeutung dieses Konstrukts (vgl. Zacher, 2015a). Wir betrachten SAW als einen Entwicklungsprozess, der in allen Karrierephasen stattfinden kann und durch Individuelle Ergebnisse wie verbesserte WA und Wohlbefinden erfasst werden kann. Wir operationalisierten das Konstrukt SAW durch zwei Cluster von Mitarbeiterwahrnehmungen: (a) psychologische Erfahrungen von Individuen mit altersbedingtem Wachstum am Arbeitsplatz (SAW) und (b) Strategien zur Erreichung von SAW. Wachstumserfahrungen können sich auf verschiedene Bereiche wahrgenommener altersbedingter positiver Veränderungen beziehen, darunter verbessertes Wissen und Erfahrung, soziale Fähigkeiten, Emotionsregulation und Anpassungsfähigkeit in arbeitsbezogenen Situationen (vgl. Taneva et al., 2016). Strategien (oder Moderatoren) für SAW können sowohl Mitarbeiter( z. B. proaktive Selbstregulierung) als auch organisatorische (z. B. Jobdesign, formelles HRM und informelle Arbeitsvereinbarungen) 
Verhaltensweisen umfassen (vgl. Bal, 2015; Baltes \& Baltes, 1990; Kooij et al., 2014; Taneva et al., 2016; Truxillo et al., 2012).

Die anschließende empirische Analyse mit Daten aus drei aufeinanderfolgenden MTurk-Stichproben mit insgesamt 417 Mitarbeitern im organisatorischen Umfeld ergab, dass sich die Erfahrungen der Mitarbeiter mit SAW weitgehend mit der Wahrnehmung der Mitarbeiter von SAW auf individueller Ebene (d. H. Persönliche Strategien) überschnitten. Eine mögliche Erklärung könnte sein, dass SAW auch als ein Prozess der aktiven Selbstregulation beschrieben werden könnte, der von der Motivation des Individuums angetrieben wird, Eine Anpassung an Die Person-Umwelt zu verfolgen (Kooij et al., 2019). So können die Wahrnehmungen der Mitarbeiter sowohl positive altersbedingte Veränderungen als auch proaktive selbstregulierende Verhaltensweisen (persönliche Strategien) das Konstrukt SAW im Sinne eines wahrgenommenen altersbedingten Wachstums widerspiegeln (vgl. Taneva et al., 2016).

Darüber hinaus untersuchten wir die konstrukt- und kriterienbezogene Validität der neuen Skala, indem wir die Assoziationen der Gesamtpunktzahl der SAW-Skala mit den Punktzahlen von sechs etablierten Messgrößen persönlicher (SOC) und organisatorischer (Job design, formaler HRM) Strategien sowie subjektiver Kriterien für SAW (WA, gedeihen und überleben bei der Arbeit) untersuchten. Die empirischen Analysen bestätigten alle erwarteten Beziehungen, abgesehen von der Assoziation zwischen dem SAWScore und dem Surviving at Work-Score, dem es an statistischer Signifikanz zu mangeln schien. Dies könnte daran liegen, dass wir vorgeschlagen haben, bei der Arbeit als vorläufiges Kriterium für SAW zu überleben. Obwohl die Erfahrungen der Mitarbeiter mit dem Überleben bei der Arbeit auf die Erhaltung der Ressourcen und damit auf nachhaltige Arbeitsergebnisse (z. B. Wohlbefinden) hindeuten können (vgl. Kooij, 2015; Taneva \& Arnold, 2018), impliziert das Konstrukt, das bei der Arbeit überlebt, kein wahrgenommenes Wachstum, wie es bei SAW in Zachers Definition der Fall ist (vgl. Zacher, 2015a).

\section{Beiträge zu Theorie und Praxis}

Diese Studie trug in mehrfacher Hinsicht zur Theorieentwicklung bei. Erstens systemamete und konzeptualisierte es Schlüsseldomänen des entstehenden Konstrukts SAW durch die Verknüpfung von Personen- und Umweltvariablen. Damit reagierte sie auf die jüngsten Forderungen, mehrstufige Faktoren bei der Untersuchung dieses Konstrukts zu integrieren (vgl. Zacher, 2015a; Zacher et al., 2018). Zweitens konzentrierte es sich auf die Synergien zwischen diesen Domänen und gewann neue Erkenntnisse darüber, wie sie zusammen und getrennt arbeiten. Drittens übersetzte es jede Domäne in praktische Begriffe und untersuchte ihre konstrukt- und kriterienbezogene Gültigkeit in Bezug auf andere, bekannte Konstrukte und demografische Variablen. So wurden neue Informationen über den Inhalt jeder Domäne gefunden und ein neues Messgerät entwickelt. Aus Sicht der Praktiker wird dieses Instrument Aus- und Weiterbildungsfachleuten helfen, die Erfahrungen und das Potenzial der Mitarbeiter, erfolgreich in Organisationen zu altern, zu erfassen. Darüber hinaus kann diese Skala den Arbeitnehmern helfen, ihre eigenen Erfahrungen mit SAW zu überwachen und zu lenken. Dies wiederum kann organisatorische Systeme und Initiativen zur Unterstützung von SAW beeinflussen und somit das höhere Wohlbefinden und die Leistung der Mitarbeiter bei der Arbeit sicherstellen. Längerfristig kann die Unterstützung von SAW sowohl aus individueller als auch aus organisatorischer Sicht dazu beitragen, ein längeres und gesünderes Arbeitsleben zu erhalten.

\section{Grenzen und Wege für Zukünftige Forschung}

Zukünftige Forschung muss weitere Beweise für die Gültigkeit der SAW-Skala liefern. Zum Beispiel fanden wir eine signifikante positive Beziehung des Alters zur organisatorischen Amtszeit, aber wir fanden keine Hinweise auf altersbedingte Unterschiede in Bezug auf die Selbstwahrnehmung der Mitarbeiter von SAW. Der Mangel an Nachweisen altersbedingter Unterschiede kann darauf zurück zurückfallen, dass die Altersmerkmale unserer Teilnehmergruppen nicht gut genug spezifiziert wurden oder weil es im Allgemeinen kein klares, definitives Kriterium für die Einteilung von Arbeitnehmern in "älter" und "jünger" gibt (vgl. Zaniboni, Kmicinska, Truxillo, Kahn, Paladino \& Fraccaroli, 2019). Auch unsere Pilot- und Kalibrierproben enthielten keine Teilnehmer über 60 Jahre. Es kann sein, dass Menschen ab 60 Jahren, die in der Belegschaft bleiben, SAW anders erleben als Arbeitnehmer in jüngeren Jahren. Zukünftige 
Forschung sollte sich mit diesem Problem befassen. Darüber hinaus ist es möglich, dass verschiedene Moderatoren eine Rolle bei der Alterung der Arbeitnehmer spielen (vgl. Felicitas, Goecke, \& Kunze, 2016; Zacher et al., 2018). Wichtig ist, dass der Mangel an Nachweisen altersbedingter Unterschiede in der Selbstwahrnehmung der Mitarbeiter von SAW darauf hindeuten kann, dass die von uns vorgeschlagene Maßnahme SAW in allen Karrierestufen erfasst (vgl. Zacher et al., 2018).

Darüber hinaus haben wir uns in dieser Phase der Entwicklung der Skala auf mehrere Beschäftigungsmerkmale und eine kleine Reihe demografischer Variablen konzentriert. Wir haben keine Informationen über diese Arbeitsmerkmale gesammelt, die in einigen Karrierephasen (z. B. hohe körperliche Anforderungen in der späten Karriere), den Berufen, Organisationen und Industriesektoren der Teilnehmer negativ mit SAW in Verbindung gebracht werden können. Solche Informationen würden helfen, das SAW-Konstrukt und -Maß besser zu kontextualisieren und zu verstehen. Außerdem stützten wir uns auf Daten von MTurk-Arbeitern, die die Verallgemeinerbarkeit der Studienergebnisse einschränken können (vgl. Walters, Christakis \& Wright, 2018). In Zukunft sollten SAW-Forscher versuchen, Daten von Arbeitnehmern in allen Karrierephasen und verschiedenen beruflichen / industriellen / kulturellen Umgebungen mit Nicht-MTurk-Stichproben zu sammeln. Dies ermöglicht Vergleiche zwischen Gruppen von Arbeitnehmern in verschiedenen Karrierestufen/Berufen/Sektoren/Kulturen. Um die Altersdimension von SAW besser zu verstehen, sollten Die Forscher neben dem chronologischen Alter der Arbeiter auch andere Alterskonstrukte (z. B. subjektives Alter) berücksichtigen (vgl. Felicitas et al., 2016) und einen longitudinalen Ansatz verfolgen (vgl. Kooij et al., 2019). Darüber hinaus haben wir in dieser Studie die Assoziationen zwischen SAW und nur drei arbeitsbezogenen Ergebnissen (d. H. WA und psychologisches Wohlbefinden, verstanden als Gedeihen bei der Arbeit und Überleben bei der Arbeit) erfasst, die sich alle selbst bewerteten. Daten zu anderen Arbeitsergebnissen (z. B. Leistung, Gesundheit, andere Formen des Wohlbefindens, Einstellungen zur Arbeit, Arbeitsmotivation, Erwartungen usw.; vgl. Zacher, 2015a) und aus mehreren Quellen werden in der zukünftigen Forschung mit der SAW-Skala am wertvollsten sein.

\section{TRANSLATED VERSION: PORTUGUESE}

Below is a rough translation of the insights presented above. This was done to give a general understanding of the ideas presented in the paper. Please excuse any grammatical mistakes and do not hold the original authors responsible for these mistakes.

\section{VERSÃO TRADUZIDA: PORTUGUÊS}

Aqui está uma tradução aproximada das ideias acima apresentadas. Isto foi feito para dar uma compreensão geral das ideias apresentadas no documento. Por favor, desculpe todos os erros gramaticais e não responsacule os autores originais responsáveis por estes erros.

\section{INTRODUÇÃO}

O conceito emergente de envelhecimento bem-sucedido no trabalho (SAW) está enraizado nas interpretações contemporâneas das teorias de desenvolvimento organizacionais e de vida. Enfatiza o importante papel dos locais de trabalho para o envelhecimento bem-sucedido (Olson \& Schultz, 2019), implicando que experiências longas e positivas relacionadas ao trabalho podem ser sustentadas por meio de adaptação bem-sucedida tanto intra-individual (por exemplo, relacionada à idade) quanto externas (por exemplo, ambientais) (Kooij, 2015; Olson \& Schultz, 2019; Zacher, 2015a). Kooij (2015) descreveu a SAW como a manutenção sustentável da capacidade de trabalho, saúde e motivação de um indivíduo ao longo do ciclo de vida profissional. Nessa definição, o papel ativo do colaborador para a criação de recursos de trabalho e a garantia de um ajuste presencial contínuo é de fundamental importância. Zacher (2015a, b) apontou que a SAW não se limita a manter os níveis atuais de funcionamento e pode se referir ao desenvolvimento ou crescimento de um trabalhador. Assim, a SAW pode ser vista como uma trajetória intra-individual relacionada à idade de um desfecho de trabalho (por exemplo, bem-estar ou desempenho 
no trabalho) que se desvia positivamente da trajetória média relacionada à idade. Mesmo um funcionário que experimentou um declínio em um resultado de trabalho ao longo do tempo pode estar envelhecendo com sucesso no trabalho quando sua trajetória é mais positiva do que a trajetória média relacionada à idade (Zacher, 2015a, b).

De forma sistemática, Zacher, Kooij e Beier (2018) descreveram os fatores que contribuíram para o envelhecimento ativo no trabalho (AAW), que é uma construção que se sobrepõe um pouco com a SAW (cf. Zacher, 2015a). A AAW é descrita como a capacidade dos trabalhadores de "manter ou melhorar (1) seu bem-estar físico, mental e social; (2) continuar a mostrar altos níveis de engajamento e desempenho no trabalho; e (3) experimentar tratamento justo e segurança no emprego" no processo de envelhecimento (Zacher et al., 2018, p. 37). Como o desenvolvimento é um processo ao longo da vida, a AAW refere-se a trabalhadores de todas as idades e estágios de carreira. Fatores multinível (individual, equipe, organizacional e social mais ampla) contribuem para a AAW/SAW (ver também Kooij, Zacher, Wang e Heckhausen, 2019). A ideia de compreender a AAW/SAW em relação tanto aos fatores internos (por exemplo, pessoa) quanto externos (por exemplo, ambientais) está em consonância com a crítica contínua de que, sendo focada principalmente no processo de desenvolvimento intra-individual, teorias de vida em geral provavelmente negligenciam os efeitos do contexto sobre o desenvolvimento do indivíduo (Olson \& Schultz, 2019).

Dado o crescente interesse pela AAW/SAW a partir de uma perspectiva de vida contemporânea (Zacher et al., 2018), é surpreendente que nenhuma medida dessa construção tenha sido desenvolvida ainda. Em dois estudos consecutivos em 2006 e 2007, Robson e seus colegas conceituaram critérios provisórios para o envelhecimento bem-sucedido no local de trabalho e desenvolveram um inventário para mensuração deles (Robson \& Hansson, 2007; Robson, Hansson, Abalos, \& Booth, 2006). Zacher \& Rudolph (2017) criticaram esse modelo por negligenciar os fatores contextuais e os resultados do trabalho, focando inteiramente em fatores de pessoa (estratégias comportamentais). Além disso, Zacher \& Rudolph (2017) concluíram que, por causa das estratégias comportamentais sugeridas por Robson et al. (Robson \& Hansson, 2007; Robson et al., 2006) não interagem com a idade, essas estratégias não podem ser consideradas estratégias de envelhecimento bem sucedidas. Mais recentemente, Kooij e colegas reconheceram a dificuldade de operacionalizar e, assim, medir a construção SAW (Kooij et al., 2019). Assim como zacher (2015a), entendemos a SAW como um processo de crescimento intra-individual multidimensional, que está relacionado à idade, associado a vários critérios subjetivos e objetivos, podendo ser facilitado (ou constrangido) por fatores multinível. Com base na definição de Saw (2015a) de Zacher e no quadro de fatores AAW por Zacher et al. (2018), o objetivo do nosso estudo é ampliar o conhecimento do SAW por: (a) operacionalizar importantes facetas da SAW construída e (b) criar uma única medida de SAW. Focamos nas experiências individuais e nos resultados da SAW, bem como nos antecedentes individuais e ambientais da SAW no contexto organizacional. Também consideramos as interações potenciais desses fatores com a idade dos trabalhadores (cf. Zacher et al., 2015a, 2018). Testamos as propriedades psicométricas da nova medida em três amostras consecutivas de MTurk e sugerimos direções para futuras pesquisas.

\section{Operacionalizando o envelhecimento bem-sucedido no trabalho}

Experiências dos Funcionários de SAW

Os impactos das mudanças relacionadas à idade nos comportamentos e resultados do trabalho (especialmente no final da carreira) estão amplamente documentados. A natureza e a extensão das alterações relacionadas à idade podem variar significativamente entre os indivíduos devido a diferenças de fatores corporais e ambientais. Essas mudanças (por exemplo, nas habilidades cognitivas, personalidade e motivação dos trabalhadores) provavelmente refletirão várias perdas e ganhos de desenvolvimento ao longo do ciclo de vida profissional (Kanfer \& Ackerman, 2004; Warr, 2001). Recentemente, o número de estudos focados nos aspectos positivos (ou seja, ganhos de desenvolvimento) em oposição aos aspectos relacionados à perda (ou seja, declínios do desenvolvimento) do processo de envelhecimento aumentou. Por exemplo, em um estudo qualitativo com 37 trabalhadores mais velhos (com 55 anos ou mais) de dois países (Bulgária e Reino Unido) e dois setores industriais (tecnologias de saúde e informação e comunicação), Taneva, Arnold e Nicolson (2016) descobriram que os trabalhadores de carreira tardia estão 
bem cientes de como as mudanças relacionadas à idade podem afetar sua vida profissional e resultados. É importante ressaltar que os trabalhadores associam algumas dessas mudanças (por exemplo, maior conhecimento e experiência, maior experiência social e emocional) com seu desenvolvimento pessoal e profissional e, em última instância, com melhores resultados no trabalho. Assim, é provável que as percepções dos trabalhadores sobre mudanças positivas relacionadas à idade (ganhos de desenvolvimento; cf. Kanfer \& Ackerman, 2004) indiquem as experiências dos trabalhadores com a SAW.

No estudo de Taneva et al.(2016), juntamente com suas percepções de maior conhecimento e experiência, os trabalhadores (mais velhos) comunicaram percepções de outras áreas de crescimento pessoal e profissional, entre as quais aumento da autoconsciência e confiança, melhor habilidade social, regulação das emoções, capacidade de resolver problemas complexos e adaptação geral às mudanças contextuais. Esses achados, extraídos das narrativas dos trabalhadores, correspondem aos resultados de pesquisas anteriores na tradição da vida útil (por exemplo, Carstensen et al., 2011; Kanfer \& Ackerman, 2004). Sugerimos que as conceituações dos trabalhadores sobre mudanças intra-individuais positivas possam moldar um domínio importante da SERRA de construção.

\section{Estratégias Pessoais e Organizacionais Para Saw}

Do ponto de vista da teoria da vida, o envelhecimento é um processo proativo de adaptação, impulsionado pela auto-regulação na forma de estratégias de gestão da vida que os indivíduos aplicam para lidar com mudanças relacionadas à idade (por exemplo, perda, ganho e/ ou reorganização de recursos) em si, bem como mudanças em seu ambiente (Kanfer \& Ackerman, 2004). O modelo de otimização seletiva com remuneração (SOC) (Baltes \& Baltes, 1990) propõe que os indivíduos provavelmente usem estratégias que se enquadram em três categorias de processos de autorregulação (seleção, otimização e compensação) em suas tentativas de se adaptar a mudanças relacionadas à idade e mudanças em seu ambiente. Esses comportamentos proativos tornam-se mais importantes com o aumento da idade e podem ser particularmente úteis no local de trabalho. A partir do pioneirismo de Abraão e Hansson (1995), uma infinidade de estudos tem demonstrado os efeitos positivos das estratégias de SOC sobre o bem-estar e o desempenho dos trabalhadores (por exemplo, Moghimi, Scheibe e Freund, 2019; Moghimi, Zacher, Shreible, \& Van Yperen, 2017). Assim, as estratégias de SOC podem ser um mecanismo eficaz para alcançar a SAW.

Combinando as vantagens do design de trabalho e teorias da vida útil, Truxillo, Cádiz, Rineer, Zaniboni e Fraccaroli, (2012) propuseram potenciais efeitos relacionados à idade de três subgrupos de características do trabalho (tarefa, conhecimento e social) no engajamento no trabalho, satisfação no trabalho e desempenho no trabalho. Por exemplo, eles sugeriram que, devido a mudanças intra-individuais relacionadas à idade, algumas características do trabalho, como autonomia do trabalho, significância de tarefas, variedade de habilidades e apoio social podem ser preferidas por trabalhadores mais velhos (em comparação com os mais jovens) e, portanto, facilitar resultados positivos do trabalho, particularmente no final da carreira. Entre outros, Zacher et al. (2018) enfatizaram a importância da autonomia do trabalho, da incrusidade social e do senso de sentido para os trabalhadores idosos. Portanto, permitir oportunidades para maior autonomia de trabalho, significância de tarefas, variedade de habilidades e apoio social pode fomentar a SAW em toda a vida útil (veja também Cadiz, Rineer e Truxillo, 2019).

Tanto as abordagens formais (por exemplo, Gestão Estratégica de Recursos Humanos) quanto informais (por exemplo, acordos entre funcionários e seus supervisores) de gestão de recursos humanos (HRM) são consideradas importantes para apoiar o bem-estar e o desempenho dos trabalhadores (Bal, 2015; Kooij, Jansen, Dikkers, \& De Lange, 2014). Algumas práticas formais de HRM (também referidas como "amigáveis à idade", cf. Zacher et al., 2018) são vistas como tendo efeitos desejáveis especialmente para os trabalhadores mais velhos. Kooij et al. (2014) conceituaram quatro pacotes de práticas de HRM para trabalhadores idosos, entre eles estão práticas de manutenção e desenvolvimento. Taneva e Arnold (2018) relataram que uma combinação específica entre algumas práticas de manutenção e desenvolvimento do HRM previu experiências de alto bem-estar no trabalho (prosperando no trabalho) e alto desempenho no trabalho em uma grande amostra de trabalhadores mais velhos no Reino Unido. Essas práticas, mais valorizadas pelos trabalhadores mais velhos, referem-se ao acesso ao treinamento, reconhecimento e 
respeito, atribuições significativas, feedback do supervisor, trabalho flexível e oportunidades de trabalhar por mais tempo. Além disso, há evidências consideráveis sobre o valor dos arranjos informais de trabalho e o papel dos supervisores no final da carreira. Por exemplo, Bal (2015) concluiu que "a individualização dos arranjos de carreira será cada vez mais importante na sustentabilidade das carreiras contemporâneas" (Bal, 2015, p. 1). Com base nessas evidências, sugerimos que o acesso dos trabalhadores a uma combinação de certas práticas de HRM com acordos informais de trabalho (i-deals) pode ser fundamental para a obtenção de SAW nas organizações.

\section{Resultados do Envelhecimento Bem-Sucedido no Trabalho}

Teoricamente, a SAW tem sido positivamente associada a vários desfechos de trabalho em nível individual, entre os quais motivação do trabalho, desempenho do trabalho, saúde e bem-estar (Zacher, 2015a). Até agora, apenas alguns estudos forneceram apoio empírico para algumas dessas associações. Por exemplo, Taneva e Arnold (2018) relataram três caminhos para a SAW em organizações que demonstram o papel de estratégias pessoais (SOC) e organizacionais (HRM) para alcançar alto desempenho no trabalho. Além disso, descobriram que o bem-estar psicológico dos colaboradores mediava as relações de estratégias pessoais e organizacionais com desempenho autoavaado no trabalho (proatividade da tarefa, desempenho extra e no papel). Portanto, o bem-estar dos trabalhadores é importante e, talvez mais direto (comparado ao desempenho do trabalho), resultado da SAW.

Prosperar no trabalho é descrito como "o senso conjunto de vitalidade e aprendizado, que comunica um senso de progresso ou movimento para a frente no autodesenvolvimento" (Spreitzer, Sutcliffe, Dutton, Sonenshein, \& Grant, 2005, p. 538). Experiências de prosperidade no trabalho podem ser impulsionadas por fatores pessoais e organizacionais (Spreizer, Porath, \& Gibson, 2012). Prosperar no trabalho está positivamente relacionado a resultados como alto desempenho no trabalho e na carreira, comportamentos positivos no trabalho, relacionado ao trabalho e bem-estar geral (Porath, Spreitzer, Gibson, \& Garnett, 2012). Dois estudos de Taneva e colegas (Taneva et al., 2016; Taneva \& Arnold, 2018) demonstrou que altos níveis de prosperidade no trabalho também são comuns entre os trabalhadores mais velhos. Assim, experiências de prosperidade no trabalho podem acontecer em todas as etapas da carreira. Além disso, prosperar no trabalho pode ser visto tanto como uma forma de bem-estar psicológico (cf. Spreitzer et al., 2005; Warr, 2007) e um critério para SAW (Taneva \& Arnold, 2018).

Ao contrário de prosperar, a construção emergente de sobreviver no trabalho é descrita como a tendência do indivíduo de preservar os recursos físicos e mentais, limitando suas atividades de trabalho e perspectivas para lidar com condições de trabalho que são percebidas como altamente exigentes (Taneva et al., 2016). Tal experiência de preservação pode indicar níveis reduzidos ou falta de SAW. No entanto, Taneva e Arnold (2018) descobriram que, pelo menos em algumas circunstâncias, as experiências de sobrevivência no trabalho podem ser de fato um sinal de forma sustentada da pessoa-ambiente e, em última instância, da SAW. Isso também está em linha com a definição de Kooij de SAW como manutenção sustentável (Kooij, 2015) e a definição de AAW por Zacker e colegas (Zacher et al., 2018). Portanto, sobreviver no trabalho pode ser outro indicador de SAW.

Além disso, conforme descrito nas seções anteriores, as experiências individuais de SAW podem ser aprimoradas utilizando estratégias pessoais (por exemplo, SOC) ou organizacionais (por exemplo, HRM, i-deals e job design) que facilitam a regulação de seus recursos. O conceito de capacidade de trabalho (WA) descreve o equilíbrio (ajuste) entre o trabalho e os recursos pessoais (Tuomi, Ilmarinen, Jahkola, Katajarinne, \& Tulkki, 1998). Pesquisas recentes de vida têm demonstrado que certos trabalhos (por exemplo, controle de emprego, feedback e apoio social) e características de pessoas (por exemplo, comportamentos proativos) podem apoiar a manutenção e até mesmo o aumento da WA, especialmente no

final da carreira (por exemplo, Weigl, Müller, Hornung, Zacher, \& Angerer, 2013). Neste contexto, foi sugerido que a WA pode ser um critério útil para a SAW no final da carreira (e além) (Kooij, 2015; Weigl et al., 2013). 


\section{CONCLUSÃO}

Desenvolvemos e estabelecemos as propriedades psicométricas da primeira medida única para SAW no significado contemporâneo desta construção (cf. Zacher, 2015a). Vemos o SAW como um processo de desenvolvimento que pode ocorrer em todas as etapas da carreira e pode ser capturado através de resultados individuais, como melhor WA e bem-estar. Operacionalizamos a SERRA de construção através de dois clusters de percepções dos colaboradores: (a) experiências psicológicas dos indivíduos de crescimento relacionado à idade no local de trabalho (SAW) e (b) Estratégias para alcançar a SAW. As experiências de crescimento podem se referir a diversas áreas de mudanças positivas relacionadas à idade percebida, entre as quais estão conhecimento e experiência aprimorados, habilidades sociais, regulação das emoções e adaptabilidade em situações relacionadas ao trabalho (cf. Taneva et al., 2016). As estratégias (ou facilitadores) para a SAW podem incluir comportamentos tanto de funcionários (por exemplo, autoregulação proativa) quanto organizacionais (por exemplo, desenho de trabalho, HRM formal e arranjos informais de trabalho) (cf. Bal, 2015; Baltes \& Baltes, 1990; Kooij et al., 2014; Taneva et al., 2016; Truxillo et al., 2012).

Análises empíricas subsequentes com dados de três amostras consecutivas de MTurk com 417 trabalhadores em ambiente organizacional revelaram que as experiências dos funcionários de SAW se sobrepuseram em grande parte com as percepções dos funcionários sobre os facilitadores de nível individual da SAW (ou seja, estratégias pessoais). Uma explicação potencial pode ser que o SAW também possa ser descrito como um processo de auto-regulação ativa, impulsionado pela motivação do indivíduo para buscar o ajuste do ambiente da pessoa (Kooij et al., 2019). Assim, a percepção dos colaboradores sobre mudanças positivas relacionadas à idade e comportamentos proativos de auto-regulação (estratégias pessoais) pode refletir a vivagem saw no sentido de crescimento percebido relacionado à idade (cf. Taneva et al., 2016).

Além disso, examinamos a validade da nova escala, explorando as associações da pontuação total da escala SAW com os escores de seis medidas estabelecidas de estratégias pessoais (SOC) e organizacionais (job design, formal HRM), bem como critérios subjetivos para SAW (WA, prosperando e sobrevivendo no trabalho). As análises empíricas confirmaram todas as relações antecipadas, além da associação entre o escore da SAW e o escore Sobrevivente no Trabalho, que parecia não ter significância estatística. Isso pode ser porque sugerimos sobreviver no trabalho como um critério provisório para a SAW. Embora as experiências dos colaboradores de sobreviver no trabalho possam indicar manutenção de recursos e, portanto, resultados sustentados do trabalho (por exemplo, bem-estar) (cf. Kooij, 2015; Taneva \& Arnold, 2018), a construção que sobrevive no trabalho não implica crescimento percebido como é o caso da SAW na definição de Zacher (cf. Zacher, 2015a).

\section{Contribuições para a Teoria e a Prática}

Este estudo contribuiu para o desenvolvimento teórico de várias maneiras. Primeiro, sistematizou e conceituou ainda mais domínios-chave do SERRA de construção emergente, ligando variáveis de pessoa e meio ambiente. Assim, respondeu aos recentes apelos para a integração de fatores multinível ao examinar essa construção (cf. Zacher, 2015a; Zacher et al., 2018). Em segundo lugar, concentrou-se nas sinergias entre esses domínios e ganhou novas percepções sobre como eles operam juntos, bem como separadamente. Em terceiro lugar, traduziu-se em termos práticos de cada domínio e explorou sua validade de construção e critério no que diz respeito a outros construtos conhecidos e variáveis demográficas. Assim, novas informações sobre o conteúdo de cada domínio foram encontradas e um novo instrumento de medição foi desenvolvido. Do ponto de vista do praticante, este instrumento ajudará profissionais de treinamento e desenvolvimento a captar experiências e potencialidade dos trabalhadores para envelhecer com sucesso nas organizações. Além disso, essa escala pode ajudar os trabalhadores a monitorar e direcionar suas próprias experiências de SAW. Isso, por sua vez, pode informar sistemas e iniciativas organizacionais com o objetivo de apoiar a SAW e, portanto, garantir maior bem-estar e desempenho do trabalho dos colaboradores. A longo prazo, apoiar a SAW tanto do ponto de vista individual quanto organizacional pode ajudar a sustentar uma vida profissional mais longa e saudável. 


\section{Limitações e Caminhos para Pesquisas Futuras}

Pesquisas futuras precisam fornecer mais evidências da validade da escala SAW. Por exemplo, encontramos uma relação positiva significativa da idade com a posse organizacional, mas não encontramos evidências de diferenças relacionadas à idade em relação à autopercepção dos funcionários sobre a SAW. A falta de evidência de diferenças relacionadas à idade pode ser porque as características etárias de nossos grupos participantes não foram especificadas o suficiente ou porque geralmente não há um critério claro e definitivo para dividir os trabalhadores em "mais velhos" e "mais jovens" (cf. Zaniboni, Kmicinska, Truxillo, Kahn, Paladino, \& Fraccaroli, 2019). Além disso, nossas amostras de piloto e calibração não incluíram nenhum participante com mais de 60 anos de idade. Pode ser o caso de pessoas com 60 anos ou mais, que permanecem na força de trabalho, experimentarem a SAW de forma diferente em comparação com os trabalhadores em idades mais jovens. Pesquisas futuras devem abordar essa questão. Além disso, é possível que vários moderadores desempenham um papel no envelhecimento dos trabalhadores (cf. Felicitas, Goecke, \& Kunze, 2016; Zacher et al., 2018). É importante ressaltar que a falta de evidências de diferenças relacionadas à idade nas autoperpercepçãos da SAW dos funcionários pode indicar que a medida, que sugerimos, captura a SAW em todas as etapas da carreira (cf. Zacher et al., 2018).

Além disso, nesta fase do desenvolvimento da escala focamos em diversas características de trabalho e uma pequena gama de variáveis demográficas. Não coletamos informações sobre essas características de trabalho, que podem estar negativamente associadas à SAW em algumas etapas de carreira (por exemplo, altas demandas físicas no final da carreira), ocupações, organizações e setores industriais dos participantes. Tais informações ajudariam a contextualizar e entender melhor a construção e a medida do SAW. Além disso, baseamos-se em dados dos trabalhadores da MTurk, que podem limitar a generalizabilidade dos resultados do estudo (cf. Walters, Christakis, \& Wright, 2018). No futuro, os pesquisadores da SAW devem procurar coletar dados de trabalhadores em todas as etapas da carreira e em diversos ambientes ocupacionais/industriais/culturais com amostras não MTurk. Isso permitirá comparações entre grupos de trabalhadores em diferentes estágios/ocupações/setores/culturas. Para entender melhor a dimensão etária da SAW, os investigadores devem considerar outras construções etárias (por exemplo, idade subjetiva) além da idade cronológica dos trabalhadores (cf. Felicitas et al., 2016) e seguir uma abordagem longitudinal (cf. Kooij et al., 2019). Além disso, neste estudo captamos as associações entre a SAW e apenas três desfechos relacionados ao trabalho (ou seja, WA e bem-estar psicológico, entendidos como ambos prosperando no trabalho e sobrevivendo no trabalho), todos os quais autoavacorados. Dados relativos a outros desfechos do trabalho (por exemplo, desempenho dos trabalhadores, saúde, outras formas de bem-estar, atitudes de trabalho, motivação do trabalho, expectativas, etc.; cf. Zacher, 2015a) e de múltiplas fontes serão mais valiosos em pesquisas futuras com a escala saw. 